\title{
CHANNEL CODING FOR SATELLITE MOBILE CHANNELS
}

\author{
K. H. H. WONG, L. HANZO AND R. STEELE \\ Department of Electronics and Computer Science, University of Southampton, Southampton SO9 5NH, U.K.
}

\begin{abstract}
SUMMARY
The deployment of channel coding and interleaving to enhance the bit-error performance of a satellite mobile radio channel is addressed for speech and data transmissions. Different convolutional codes (CC) using Viterbi decoding with soft decision are examined with inter-block interleaving. Reed-Solomon (RS) codes with Berlekamp-Massey hard decision decoding or soft decision trellis decoding combined with block interleaving are also investigated. A concatenated arrangement employing RS and CC coding as the outer and inner coders, respectively, is used for transmissions via minimum shift keying (MSK) over Gaussian and Rayleigh fading channels. For an interblock interleaving period of 2880 bits, a concatenated arrangement of an $\mathrm{RS}(48,36)$, over the Galois field $\mathrm{GF}(256)$ and punctured $\operatorname{PCC}(3,1,7)$ yielding an overall coding rate of $1 / 2$, provides a coding gain of $42 \mathrm{~dB}$ for a BER of $10^{-6}$, and an uncorrectable error detection probability of $1-10^{-9}$.
\end{abstract}

KEY wORDS Channel coding Mobile satellite channels Convolutional coding Reed-Solomon coding Interleaving Trellis decoding of block codes

\section{INTRODUCTION}

In this age of information technology it is becoming essential to communicate information to people who are on the move, and may be difficult to locate precisely. The nineteenth century concept of a fixed telephone network that requires its customers to sit near their telephones in order to receive calls is antiquated. Cellular mobile radio systems are being deployed world-wide at a rapid rate and a new communications network is evolving whereby the fixed network enables communications between users having mobile terminals. ${ }^{1-3}$ The urban, and indeed rural, services are best served by terrestrial systems, with cell sizes having dimensions from $100 \mathrm{~m}$ to $5 \mathrm{~km}$. These small cells are essential to accommodate vast numbers of mobile users.

Less populated areas, including the vast oceans and seas, can be efficiently handled by mobile satellite communications. At the present time the INMARSAT $^{4}$ system having three geostationary satellites above the Atlantic, Pacific and Indian Oceans provide global coverage, except over the polar regions. The system can support three types of terminal services which are designated as Standard A, B and C. The Standard-A service has been approved, and ship earth-stations (SES) operate with a $1.0 \mathrm{~m}$ diameter stabilized $23 \mathrm{~dB}$ gain parabolic antenna. The communication capabilities include analogue FM voice, telex, facsimile and data transmission rates up to $56 \mathrm{~kb} / \mathrm{s}$. The Standard-B service is the future extension of Standard-A and is expected to be introduced in the early 1990s. It is designed to be smaller and less expensive than the StandardA equipment and will appeal to users of small vessels, such as fishing boats. It will provide digital voice at $16 \mathrm{~kb} / \mathrm{s}$, telex, data and telematic services. Recently deployed to cover low bit-rate services, the Standard- $C$ terminal operates with an antenna that is sufficiently small (about $15 \mathrm{~cm}$ ) for it to be mounted on a small boat for maritime communications, or to be attached to cars for land mobile communications. It provides telex and low-speed data transmission at rates up to $600 \mathrm{~b} / \mathrm{s}$.

In satellite mobile communications the down-link channels are usually more hostile than the up-link ones because of the shadowing effects and the multipath scatterings from objects close to the earth mobile station. The carrier frequencies used on the down-links of the maritime mobile channels ${ }^{5}$ of the INMARSAT are in the 1.530 to $1.545 \mathrm{GHz}$ band. The coherence bandwidth for these transmissions is greater than $100 \mathrm{kHz}$, and hence the time-varying multipath fading channel can be assumed to have non-frequency-selective properties for narrowband data transmissions at $16 \mathrm{~kb} / \mathrm{s}$. In the case of maritime communications the multipath fading arises from signal reflections from the sea's surface, shadowing effects due to the ship's superstructure and Doppler offset due to the ship's motion. The channel can be characterized by a Rician fading model with a direct signal power $C$ and a diffuse scattered signal of mean power $M$. The Rician parameter $C / M$ at the edge of the satellite coverage with the satellite operating with a $5^{\circ}$ elevation angle is $8-9 \mathrm{~dB}$ and this $C / M$ decreases with low elevation until no dominant signal is observed, whence the Rayleigh fading model is applicable. Similar comments can be made regarding land satellite mobile communi- 
cations, as found with the MARECS A satellite ${ }^{7}$ operating at $1.54 \mathrm{GHz}$ in $L$-band.

The Rician channels associated with mobile satellite communications approach either a Gaussian or a Rayleigh fading channel depending on $C / M$, and consequently we consider both of these channels when designing mobile satellite data transmission links. We note that the Rayleigh fading channel, characterized by deep fades, can by judicious choice of interleavers be rendered into a near look-alike Gaussian channel. This is because interleaving the data prior to transmission, and de-interleaving at the receiver, enables the Rayleigh-fading-induced error bursts to be dispersed. To accommodate the error bursts the forward error correction (FEC) must have sufficiently long memory and appropriately powerful error correcting capability. Long codes are preferable because their higher error averaging capability enables both random and bursty errors to be corrected. By employing long codes the number of errors in the block is, to a first approximation, independent of the channel behaviour. On the other hand, long codes imply a long coding delay which might be unacceptable in a system context. We may therefore view the same satellite mobile channel quite differently depending on the FEC employed. A very high integrity channel for the transmission of computer data may be realized by increasing the system delay. However, for speech communications this delay must be considerably lower. In the case of block codes the coding delay can easily be adjusted to any arbitrary value, but for convolutional codes the delay arises from the necessity of employing interleavers to disperse the error bursts. In our deliberations we explore effective ways of signalling over satellite channels. Theoretical and simulation results are presented for both Guassian and Rayleigh fading channels as they constitute the best and worst types of satellite mobile environments.

Figure 1 shows the system block diagram used in our studies. Two layers of codes are concatenated and two interleavers are used to combat the error bursts. The strategy is for an inner FEC decoder to correct the random errors, leaving the outer decoder to remove the error bursts that they may arise from the channel, or may have been induced due to the over-loading of the inner decoder. The combined inner and outer channel coding rate is selected to be half-rate to provide good error correcting

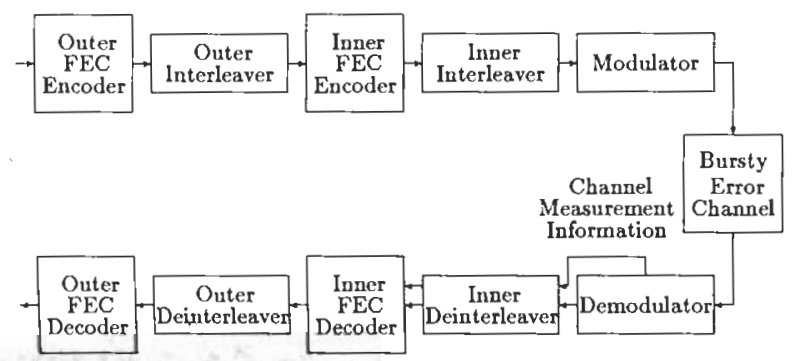

Figure 1. System block diagram capability in both FEC layers and an acceptable channel throughput. This rate is also used for the Groupe Speciale Mobile (GSM) Pan-European mobile radio speech communication system. ${ }^{8}$ Different FEC coding and interleaving strategies are described for this rate. Minimum shift keying (MSK) modulation is employed because of its reasonable bandwidth efficiency coupled with its continuous phase transition characteristics that result in only minor envelope distortions when the modulated signal is bandlimited in a satellite repeater.

The theory of Viterbi decoding (VD) of convolutional coded (CC) signals is well documented in classical references, ${ }^{9-11}$ and the background of the Berlekamp-Massey decoding algorithm for Reed-Solomon (RS) codes is also well understood. ${ }^{12.13}$ Consequently neither will be discussed here. However, because trellis decoding of block codes is not widely known we provide in Section 2 a rudimentary description of this procedure. Emphasis is given to interleaving techniques in Section 3 as they play a prominent role in combatting the fading behaviour of the channel. In Sections 4 and 5 , we derive the theoretical results for the BER performance of CC and RS, together with simulation results that verify their validity. The final Section discusses the implications of our findings.

\section{TRELLIS DECODING OF BLOCK CODES}

In our quest for a powerful FEC coding scheme for satellite mobile communications we investigate the performance of the trellis decoding ${ }^{14}$ of both binary and non-binary block codes. We commence by considering the decoding process of the non-binary maximum separable distance subclass of block codes, namely RS codes. These codes are represented by $\operatorname{RS}(n, k)$, where $k$ information symbols are encoded into $n$ symbols. The code is defined over $\operatorname{GF}\left(q^{m}\right)$, where $q$ is a prime and $m$ is an integer. $\operatorname{An} \operatorname{RS}(n, k)$ code word containing up to $t=(n-k) / 2$ error symbols can be corrected by using the Berlekamp-Massey decoding method. For systematic codes the generator polynomial $g(z)=$ $g_{2,} z^{2 t}+\cdots+g_{1} z+g_{0}$ formulates a code word by appending $(n-k)$ parity symbols $P_{n-k-1}, \ldots, P_{1}, P_{0}$ to $k$ information symbols. The encoder employs a shift register (SR) having $(n-k)$ stages, as depicted in Figure 2.

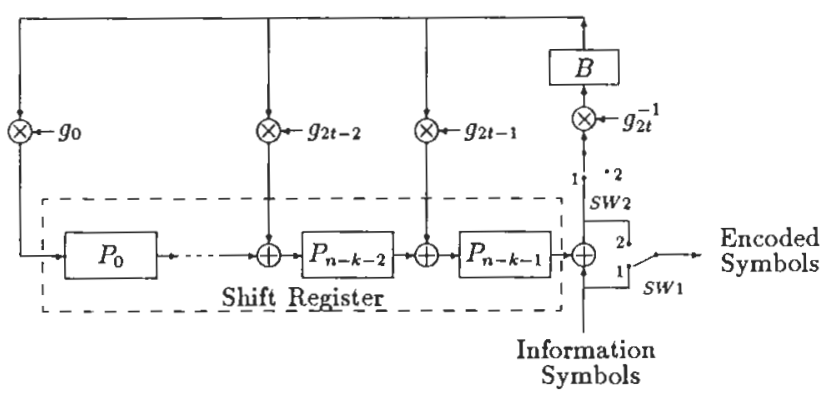

Figure 2. Systematic encoder for block codes 
At the beginning of the encoding process, the SR is cleared and both switches SW1 and SW2 are placed in position 1 . The first $k$ information symbols are passed directly to the encoder output forming the information part of the code word and they are also multiplied over $\mathrm{GF}\left(q^{m}\right)$ by the coefficient $g_{2 t}^{-1}$. This product is buffered in register $B$. The value of register $B$ is multiplied by $g_{2 t-1}$, followed by GF $\left(q^{m}\right)$ addition with $P_{n-k-2}$ to form a new parity symbol $P_{n-k-1}$. Again, the new $P_{n-k-2}$ value is calculated by multiplying the content of register $B$ by $g_{2 t-2}$ and then adding $P_{n-k-3}$ to this product. Similar multiplications and additions are performed to achieve new values from $P_{n-k-3}$ to $P_{0}$ sequentially. The second information symbol enters the encoder, and the cycle of multiplications and additions is repeated to yield a new set of $(n-k)$ parity symbol values. After the $k$ th information symbol has entered the encoder and has produced the parity symbols, the switches are turned to position 2, preventing data from entering the SR. The $n-k$ parity symbols are removed serially from the encoder.

Each parity symbol has $m$ bits, and $(n-k)$ parity symbols result in $q^{m(n-k)}$ different states in the SR. The sequential change of states during the process of encoding a code word can be catalogued as a particular path in a trellis. There are $\left(q^{m}\right)^{k}$ unique paths in the trellis and each path represents a particular code word. The trellis has $\left(q^{m}\right)^{n-k}$ rows and a depth of $n+1$ columns. The nodes on the same row represent the same state value, whereas the nodes on the same column correspond to the possible states. The state-changes between adjacent columns in the trellis are marked by the transition vector to which a symbol is attached that specifies the information symbol activating the state transition.

Initially all the parity symbols in the SR are set to their zero states. The number of encoder states increases as each new information symbol enters the encoder. The symbol-signalling instants corresponding to the column positions in the trellis are indexed by the integer $j$. On inserting the first information symbol into the encoder, $j=0, q^{m}$ different nodes are possible. The arrival of the second information symbol when $j=1$ causes the number of possible nodes to increase to $\left(q^{m}\right)^{2}$. The number of possible nodes continues to increase with $j$ until the maximum number $\left(q^{m}\right)^{n-k}$ is reached. This maximum number of states is reached when $j=n-k$, and the number of possible states is kept constant until the last information symbol to be encoded has entered the $\mathrm{SR}$ at $j=k$. At this moment, the switches SW1 and SW2 are turned to position 2 and the SR is cleared one parity symbol at a time as the symbols are removed from the encoder to leave the all-zero state in the SR. The number of possible states is thus divided by $q^{\prime n}$ at every column in the trellis merging towards the allzeros state, which is reached after clocking the encoder $(n+1)$ times.

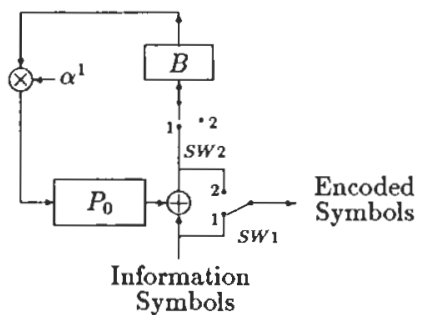

Figure 3. Systematic encoder for $\mathrm{RS}(3,2)$ over $\mathrm{GF}(4)$

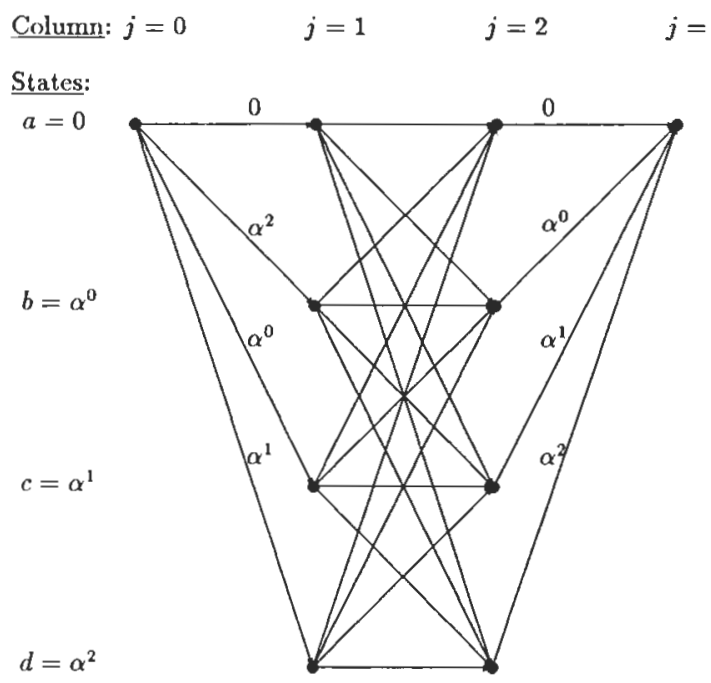

Figure 4. Trellis diagram for $\mathrm{RS}(3,2)$ over $\mathrm{GF}(4)$

Table I. Arithmetic table for GF(4) operations

\begin{tabular}{|c|c|c|c|c|c|c|c|c|c|}
\hline+ & 0 & $\alpha^{0}$ & $\alpha^{1}$ & $\alpha^{2}$ & $*$ & 0 & $\alpha^{0}$ & $\alpha^{\prime}$ & $\alpha^{2}$ \\
\hline 0 & 0 & $\alpha^{0}$ & $\alpha^{1}$ & $\alpha^{2}$ & 0 & 0 & 0 & 0 & 0 \\
\hline$\alpha^{0}$ & $\alpha^{()}$ & 0 & $\alpha^{2}$ & $\alpha^{1}$ & $\alpha^{0}$ & 0 & $\alpha^{0}$ & $\alpha^{1}$ & $\alpha^{2}$ \\
\hline$\alpha^{1}$ & $\alpha^{1}$ & $\alpha^{2}$ & 0 & $\alpha^{()}$ & $\alpha^{l}$ & 0 & $\alpha^{\prime}$ & $\alpha^{2}$ & $\alpha^{\prime}$ \\
\hline$\alpha^{2}$ & $\alpha^{2}$ & $\alpha^{l}$ & $\alpha^{0}$ & 0 & $\alpha^{2}$ & 0 & $\alpha^{2}$ & $\alpha^{0}$ & $\alpha^{1}$ \\
\hline
\end{tabular}

(a)

(b)

To aid exposition we consider the example of $\operatorname{RS}(3,2)$ code over $\mathrm{GF}\left(2^{2}\right)$ that consists of a set of four field elements $\left\{0, \alpha^{0}, \alpha^{1}, \alpha^{2}\right\}$, where $\alpha$ is the primitive element of the field. The encoding arrangement is shown in Figure 3 and its trellis diagram is displayed in Figure 4. From the basic theory of RS coding, ${ }^{13}$ the generator polynomial of this code is $g(z)=z-\alpha^{1}$, where the coefficient $g_{0}=\alpha^{1}$. This code has one parity symbol $P_{0}$ and the SR has a single stage.

The trellis contains $n+1=4$ columns $(j=$ $0,1, \ldots, 3)$ and the maximum number of states is $\left(q^{m}\right)^{n-k}=4$. The possible states are $0, \alpha^{0}, \alpha^{1}$ and $\alpha^{2}$ which are represented by the nodes $a, b, c$ and $d$, respectively. In our example, when the first information symbol $\alpha^{0}$ enters the encoder at instant $j=0$, the new state of the SR at $j=1$ is computed over GF(4) using the arithmetic operations given in Table I. 
Table II. State transition table for $\mathrm{RS}(3,2)$ code over $\mathrm{GF}(4)$

\begin{tabular}{ccccc} 
To & \multicolumn{4}{c}{ From nodes } \\
nodes & $a$ & $b$ & $c$ & \multicolumn{1}{c}{$d$} \\
\hline & & & & \\
$a$ & 0 & $\alpha^{2}$ & $\alpha^{0}$ & $\alpha^{1}$ \\
$b$ & $\alpha^{0}$ & $\alpha^{1}$ & 0 & $\alpha^{2}$ \\
$c$ & $\alpha^{1}$ & $\alpha^{0}$ & $\alpha^{2}$ & 0 \\
$d$ & $\alpha^{2}$ & 0 & $\alpha^{1}$ & $\alpha^{0}$ \\
\hline
\end{tabular}

From the encoder diagram in Figure 3 we observe that parity symbol $P_{0}$ becomes $\left(0+\alpha^{0}\right){ }^{*} \alpha^{1}=\alpha^{1}$, and this transition in the trellis diagram is shown in Figure 4. From this state $\alpha^{1}$ at $j=1$ the encoder can change at $j=2$ to any of the possible states depending on the information symbol to be encoded. The state transition in Table II provides the change of states from the current node to any other for the transition from $j=1$ to $j=2$. Thus if the next information symbol is $\alpha^{2}$, the GF(4) addition with $P_{0}$, i.e. $\alpha^{1}$ gives $\alpha^{0}$ (see Table I(a)), and on GF(4) multiplication by $\alpha^{1}$ we have $\alpha^{1}$ (see Table I(b)) and the state $c$ is reached at $j=2$. At this point SW1 and SW2 are changed to position 2 and the parity symbol $\alpha^{1}$ is removed from SR whence the encoder returns to its all-zero state.

This trellis for the $\mathrm{RS}(3,2)$ code is suitable for maximum likelihood decoding by the Viterbi algorithm ${ }^{9,10}$ to improve the BER performance. Furthermore, an improved performance can be achieved by soft-decision decoding.

\section{INTERLEAVING TECHNIQUES}

Interleaving is a process of rearranging the ordering of a sequence of symbols in some unique one-toone deterministic manner. The reverse of this process is de-interleaving, which restores the sequence to its original order. Interleaving techniques ${ }^{15}$ are generally deployed to disperse burst errors when the received signal level fades, and thereby reduce the concentration of errors that are applied to the channel decoder for correction. Before a sequence of symbols is transmitted the symbols from several code words are interleaved. When an error burst occurs the errors on de-interleaving will be shared among numerous code words that require a less powerful code to correct them. Thus interleaving effectively makes the channel appear like a random error channel to the decoder. As the interleaving period increases, the error performance can be expected to improve in the sense that noise bursts are more dispersed. On the other hand, the delay due to interleaving and de-interleaving increases. Consequently, there is always a trade-off between error performance and interleaving delay. In the forthcoming discourse we give a short introduction to some effective interleaving methods.

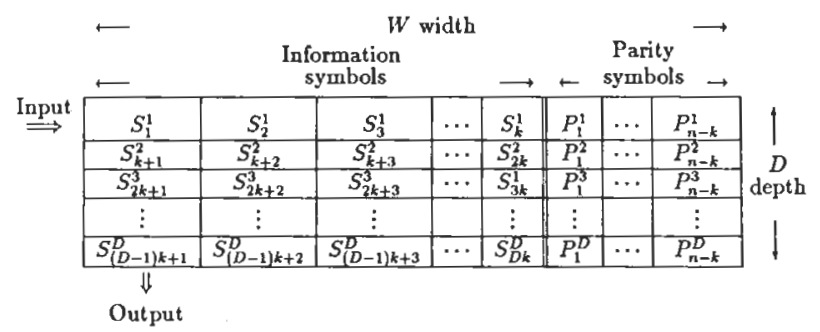

Figure 5. Block interleaver

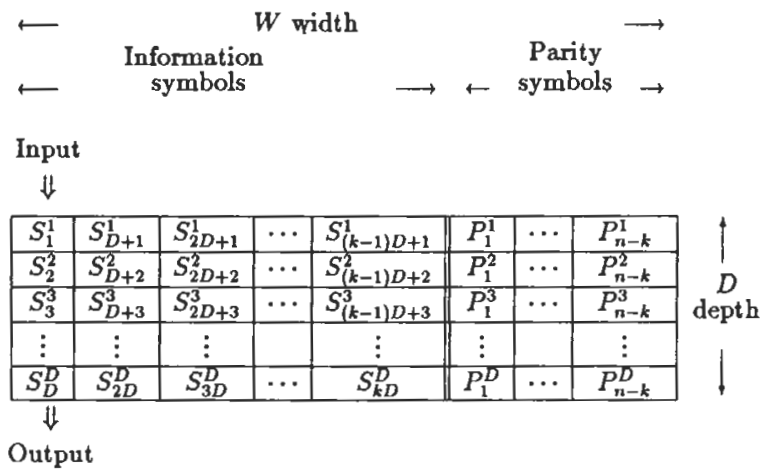

Figure 6. Modified block interleaver

\subsection{Description of interleaving techniques}

3.1.1. Block interleaving. A block interleaver takes a code word of $n$ symbols and writes them a row at a time into a matrix of depth $D$ rows and width $W$ columns, as shown in Figure 5. Suppose $W$ is equal to $n$, then a row of symbols in the interleaver corresponds to a code word composed of $k$ information symbols and $(n-k)$ parity symbols. After the matrix is completely filled, the symbols are serially removed from the matrix a colum at a time and transmitted over the channel. At the receiver, the de-interleaver performs the inverse permutation by sequentially feeding in the data a column at a time until the matrix is filled. The symbols along each row are removed and decoded. This interleaver is able to disperse any burst of errors of lengths $b \geqslant D$, as this results in no more than a single error in any code word. However, a periodic sequence of single channel errors spaced by $D$ symbols cause a single code word to be totally in error. The interleaver and de-interleaver end-toend delay is $2 W D$ symbols. The memory requirement is WD symbols in both the interleaver and deinterleaver.

Another block interleaver,${ }^{16}$ shown in Figure 6 is a derivation from the interleaver shown in Figure 5. Instead of writing rows of symbols into the matrix and appending the parity symbols to the $k$ successive information symbols, the symbols are now written a column at a time. The parity symbols are encoded from a row of $k$ information symbols each separated by $D$ symbols in their natural order.

As symbols $S_{1}^{1}, S_{2}^{2}, S_{3}^{3}, \ldots, S_{k D}^{D}$ occur, they are placed into the matrix and are also transmitted at 
the same time. By the time $S_{D+1}^{l}$ arrives, symbols $S_{1}^{1}, S_{2}^{2}, \ldots, S_{D}^{D}$ have been transmitted. When all the information symbols $S_{\mid}$to $S_{k D}^{D}$ are in the matrix and have been transmitted, the parity symbols $P_{1}^{1}$ to $P_{n-k}^{D}$ are calculated immediately following symbol $S_{k D}^{D}$ arrival in the matrix. The advantage of this interleaving scheme is that information symbols are transmitted in their natural order. Hence, the interleaver delay is negligible, and the end-to-end delay of $W D$ symbols is due to the de-interleaver.

The interleaver parameters $D$ and $W$ are selected so that all expected burst lengths are less than $D$. However, this type of interleaver lacks robustness when a periodic sequence of single errors spaced by $D$ symbols occurs. In this situation all the symbols in a row are erroneous and this overloads the channel decoder. The interleaving scheme described in the next section has the ability of dispersing bursty noise as well as periodic noise.

3.1.2. Inter-block interleaving. The inter-block interleaver takes an input block of $N B$ symbols and disperses $N$ symbols to each of the next $B$ output blocks. Consider an encoded symbol $x$ from the encoder and an output symbol $y$ from the interleaver. The mapping from the $m$ th symbol of the $i$ th coded input block to the $(j+B t)$ th interleaved symbol of the $(i+j)$ th output block is given by

$$
y(i+j, j+B t)=x(i, m)
$$

for all $i$, with $j=m \bmod B$, and $t=m \bmod N$.

An example of inter-block interleaving with $B=3$ and $N=2$ is illustrated in Figure 7 . The symbols of the three successive $i$ th, $(i+1)$ th and $(i+2)$ th coded input blocks are denoted as $a, b$ and $c$ respectively. Here, $y(i+j, j+3 t)=x(i, m)$, for all $i$, with $j=m$ $\bmod 3$, and $t=m \bmod 2$. For $m=0, y(i, 0)=$ $x(i, 0)$ and for $m=1, y(i+1,4)=x(i, 1)$, and so on.

It is noted that the successive symbols of the $i$ th input block are mapped to the next $B$ output blocks consecutively, but with the irregular offset position of $(j+B t)$ in each block. This irregular offset has the advantage of randomizing any periodic noise in the channel. In order to make sure that the mapping is a unique one-to-one operation, $B$ and $N$ cannot have a common multiple. This may place a constraint on the block size of $B N$ symbols. Disadvantages of
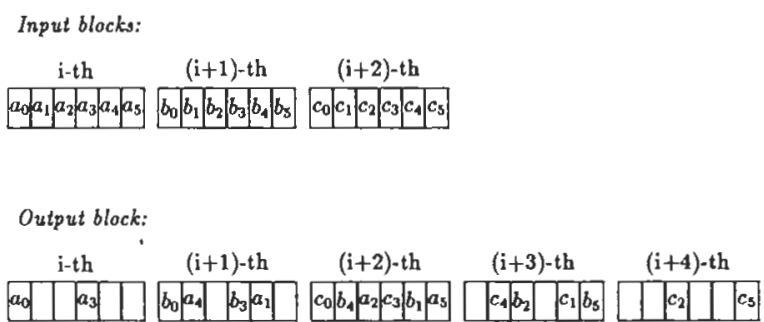

Figure 7. Example of inter-block interleaving with $B=3$ and $N=2$ this interleaving scheme are that the output sequence is expanded by $(B-1)$ blocks, and that the interleaving delay is $B^{2} N$ symbols (composed of the delay $B N$ due to buffering the input block, plus the extra delay $(B-1) B N$ due to the dispersion of the symbols).

\subsection{The impact of interleaving on channel characteristics}

The RS encoded symbols consisting of $m$ bits are serially transmitted. The receiver performs symbol regeneration prior to RS decoding. If the channel is Gaussian, or if sufficient interleaving is employed in the case of a fading channel, the probability of any bit being in error is $p_{b}$. The probability of the regenerated symbol being erroneous depends on the value of $m$. For example, Figure 8 shows the probabilities of a transmitted two bits symbol 00 being regenerated as $00,01,10$ and 11 .

In general, the probability of receiving an error symbol with $i$ bits in error is

$$
p_{\mathrm{s}, i}=\left(\begin{array}{c}
m \\
i
\end{array}\right) p_{\mathrm{b}}^{i}\left(1-p_{\mathrm{b}}\right)^{m-i}
$$

and the average symbol error probability $p$ is

$$
p_{\mathrm{s}}=\sum_{i=1}^{m}\left(\begin{array}{c}
m \\
i
\end{array}\right) p_{\mathrm{b}}^{i}\left(1-p_{\mathrm{b}}\right)^{m-i}
$$

The assumption of the memoryless channel explicit in equation (3) can be fulfilled for mobile radio channels by bit interleaving over a sufficiently long period. The delay that can be tolerated for digital speech communication restricts the interleaving period, and the assumption of memoryless channel is not always feasible.

Figure 9 shows the probability density function (PDF) of the bit errors in an 8-bit window for MSK data transmissions over Rayleigh fading channels for various bit-interleaving algorithms. Bench-mark PDFs for non-interleaving, and for the memoryless channel for which equation (3) applies are also presented. When block bit interleaving was employed the interleaving delay was 2280 bits, as $D=40$ and $W=57$, whereas for inter-block bit interleaving $B=19$ and $N=6$, giving a delay of 2166 bits, i.e. similar to that used for our block bit interleaver. Without interleaving the PDF of bit errors in a symbol was significantly different from

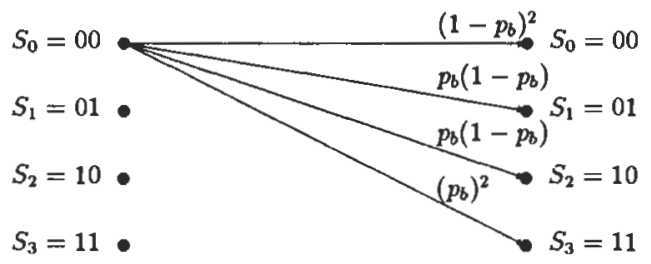

Figure 8 . Non-binary and non-symmetric channel 


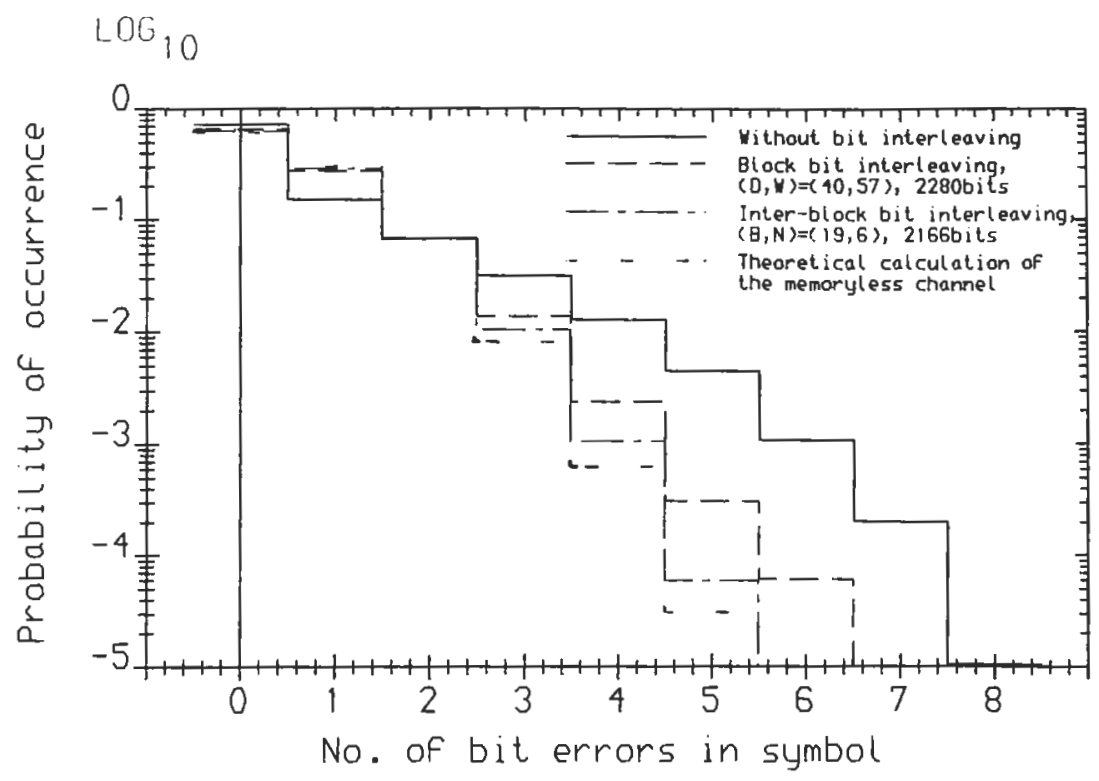

Figure 9. PDF of bit errors in 8-bit symbols over a Rayleigh fading channel with $\mathrm{SNR}=5 \mathrm{~dB}$ and $\mathrm{BER}=5.79 \times 10^{-2}$

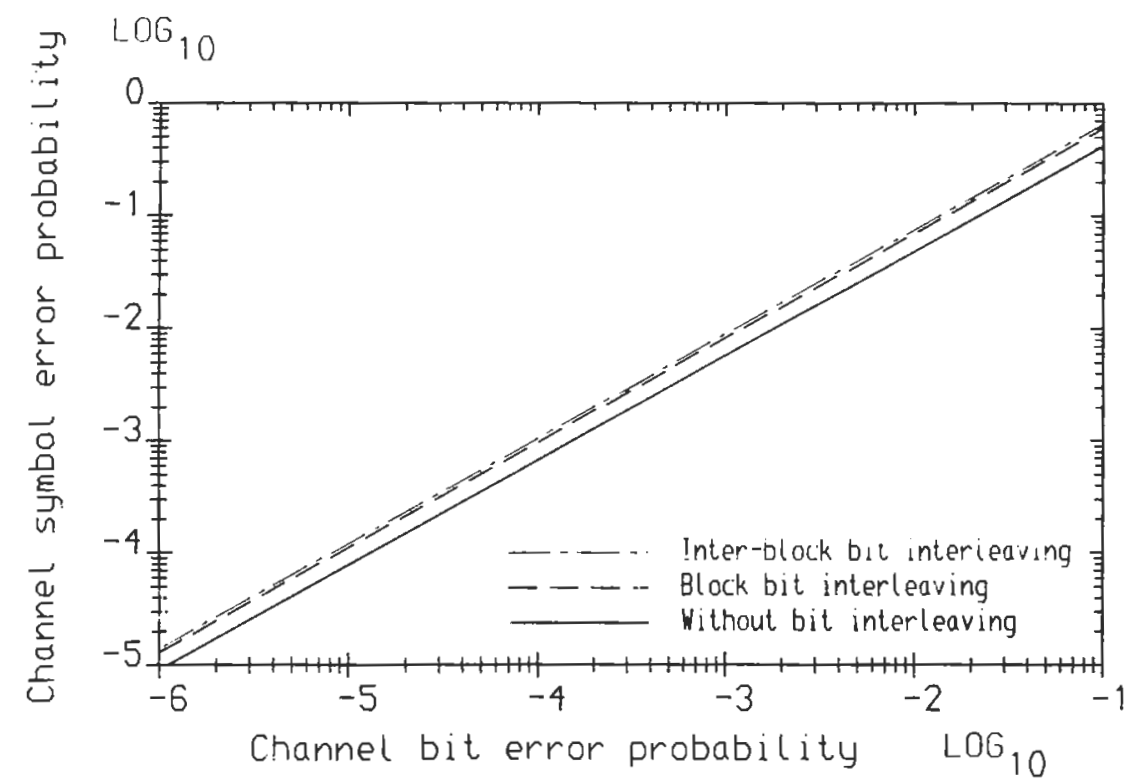

Figure 10. The effect of bit interleaving on 8-bit symbols over a Rayleigh fading channel

that of the memoryless channel. When block bit interleaving was deployed the PDF changed, indicating that the channel was beginning to approximate to a memoryless channel. By introducing the interblock bit interleaving we experienced a PDF that closely matched the theoretical curve obtained from equation (3). The experiments were repeated for different window lengths and on each occasion the inter-block interleaving yielded a PDF that was a good approximation to a memoryless channel.

Thus our experiments demonstrated that interblock bit interleaving randomized the burst errors. This process is desirable for Viterbi decoding of convolutional codes and for trellis decoding of block codes, as both decoding methods operate on bit-bybit basis. However, the random distribution of bit errors increases the symbol error probability. This is demonstrated in Figure 10, where we display simulation results of channel symbol error probability as a function of channel bit error probability for the same interleaving parameters as in Figure 9. The average number of bit errors in a symbol can be seen to be reduced as a result of interleaving due to the errors in a burst being distributed into neighbouring error-free symbols. The higher symbol error probability is undesirable for decoding methods that operate on a symbol basis, such as the Berlekamp-Massey decoding of RS codes. In this case, symbol interleaving rather than bit interleaving is preferred as it does not alter the bit error distribution within symbols, and consequently the symbol error probability is not increased. The symbol error probability $p_{\mathrm{s}}$ (see equation (3)) increases with the symbol size $m$. Simulation results 


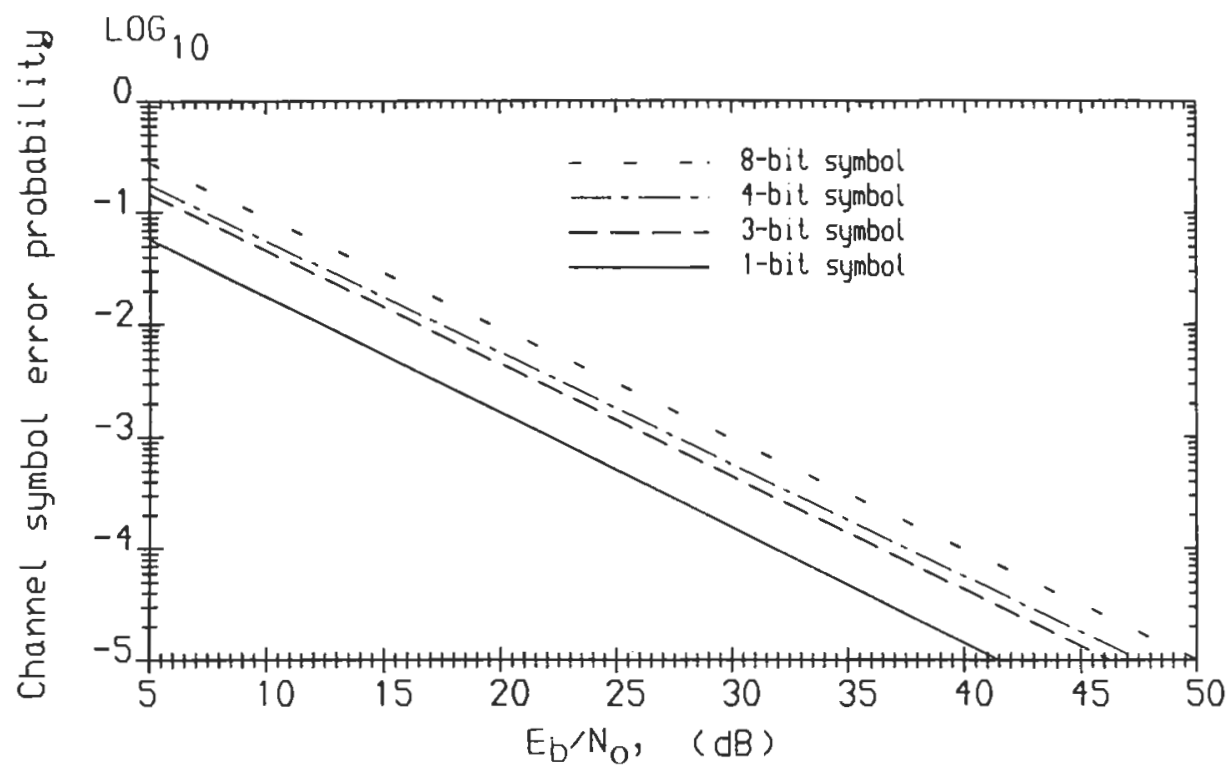

Figure 11. The effect of symbol size on symbol error probability over a Rayleigh fading channel

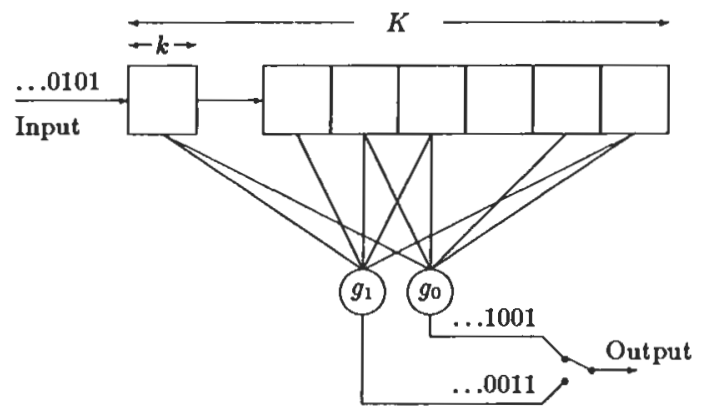

Figure 12. Convolutional encoder for the $\mathrm{CC}(2,1,7)$ code

that demonstrate this are presented in Figure 11. This increased symbol error probability discouraged the use of large symbol sizes employed in long code words of block coding.

\section{BER PERFORMANCE OF CONVOLUTIONAL CODES}

Based on an approach suggested by Viterbi, ${ }^{10}$ we now derive the transfer function of the convolutional code $\mathrm{CC}(n, k, K)$, where $k / n$ is the coding rate $R$ and $K$ is the constraint length, which is defined by the number of input stages of the encoder. Armed with this transfer function we find the weight distribution of the code and use it to determine the post-decoding error probability of the code by a union bound technique.

\subsection{BER performance of the $\mathrm{CC}(2,1,7)$ code}

The generator polynomials ${ }^{17}$ of $\mathrm{CC}(2,1,7)$ used in the encoder depicted in Figure 12 are given by and

$$
g_{1}(D)=1+D+D^{2}+D^{3}+D^{6}
$$

As the code is linear, so that the deduction of the result for this code is independent of the input data sequence, we consider the case when an allzero information data sequence is encoded and transmitted. At the receiver, if the Viterbi algorithm selects a non-zero path a decoding error ensues. As the transfer function represents the distance properties of all the possible incorrect paths, it is found by solving $2^{(K-1) k}$ state equations. For the binary code where $k=1$, the complexity of the computation grows exponentially with $K$. The alternative way of obtaining the transfer function for large values of $K$ is to trace through every possible non-zero path in the trellis by an exhaustive computer search and record the path distances. The weight distribution of code $\mathrm{CC}(2,1,7)$ can also be obtained by recording the total weight of all information sequences which produce paths of distance $d$ from the all-zero path. From our computer search we found the weight distribution to be

$$
W_{\mathrm{CC} 217}(d)=36 d^{10}+211 d^{12}+1404 d^{14}+\ldots .
$$

The coefficient $W_{10}$ of the first term in equation (5) indicates that a total of 36 information bit errors are associated with all distance $d=10$ paths. Similarly, the coefficients $W_{12}$ and $W_{14}$ of the second and third terms have 211 and 1404 information bit errors associated with distances of 12 and 14 from the all-zero path, respectively. Let the coefficient $W_{d}$ be the total number of information bit errors for paths having a distance $d$ from the all-zero path, then the minimum distance $d_{\min }$ among all of these paths is 10 , and therefore $W_{\mathrm{d}}=0$ for $d<d_{\min }$. 
The union bound on the post-decoding bit error probability $p_{\mathrm{bp}}$ for codes of rate $R$ is given by ${ }^{10}$

$$
p_{\mathrm{bp}} \leq \frac{1}{k} \sum_{d=d_{\min }}^{\infty} W_{d} P_{\mathrm{ICD}}(d)
$$

where $P_{\mathrm{ICD}}(d)$ is the probability of incorrect decoding, i.e. the probability that the decoder selects a path at distance $d$ from the correct path.

For hard-decision decoding, the decoder receives a sequence of demodulated bits having an average channel bit error probability of $p_{\mathrm{b}}$. If the number of error bits in the demodulated sequence exceeds $d / 2$ from the correct path, an incorrect path is favoured, as it has a distance of less than $d / 2$ from the demodulated sequence resulting in an incorrect decoding. The union bound of the probability of the incorrect decoding can be expressed by ${ }^{10}$

$$
P_{\mathrm{ICD}}(d)=\left\{\begin{array}{rr}
\sum_{i=(d+1) / 2}^{d}\left(\begin{array}{l}
d \\
i
\end{array}\right) p_{\mathrm{b}}^{i}\left(1-p_{\mathrm{b}}\right)^{d-i} & ; d \text { is odd } \\
\sum_{i=d / 2+1}^{d}\left(\begin{array}{c}
d \\
i
\end{array}\right) p_{\mathrm{b}}^{i}\left(1-p_{\mathrm{b}}\right)^{d-i} & \quad(7) \\
\frac{1}{2}\left(\begin{array}{c}
d \\
d / 2
\end{array}\right) p_{\mathrm{b}}^{d / 2}\left(1-p_{\mathrm{b}}\right)^{d / 2} & ; d \text { is even }
\end{array}\right.
$$

The above hard-decision decoding is independent of the modulation. Let us now consider soft-decision decoding which is modulation dependent. For MSK modulation, the demodulator output $C(T)$ at the sampling instant $T$ is ${ }^{18}$

$$
C(T)=\frac{A_{\mathrm{s}} T}{2}+N(T)
$$

where $A_{\mathrm{s}}$ is the transmitted signal amplitude, $T$ is the bit duration, $N(T)$ is a Gaussian random noise with zero mean and variance $\sigma_{N}^{2}=\eta_{0} T / 8$, and $\eta_{0} / 2$ is the double-sided power spectral density of the receiver's thermal noise. Hence $C(T)$ is also a Gaussian random variable with mean $\mu=A_{\mathrm{s}} T / 2$ and variance $\sigma^{2}=\sigma_{N}^{2}$. If the incorrect path is at a distance $d$ from the correct path, the difference in accumulated metrics occurs in those $d$ erroneous bit positions and is described by $d C(T)$ with mean equal to $d \mu$ and variance equal to $d \sigma^{2}$. The probability $P_{\mathrm{ICD}}$ of selecting the incorrect path is the probability of the metric $d C(T)$ having a negative value and is given by

$$
\begin{aligned}
P_{\mathrm{ICD}}(d) & =\frac{1}{2} \operatorname{erfc}(\sqrt{d \Gamma}) \\
& =\frac{1}{2} \operatorname{erfc}\left(\sqrt{d R \frac{E_{\mathrm{b}}}{\eta_{\mathrm{o}}}}\right)
\end{aligned}
$$

where $\Gamma=A_{s}^{2} T / \eta_{0}$ is the channel signal-to-noise ratio (SNR) and $E_{\mathrm{b}}$ is the energy per information bit. The post-decoding bit error probability for softdecision demodulation is obtained by substituting equation (9) into equation (6).

\subsection{BER performance of the punctured $\operatorname{PCC}(3,1,7)$ code}

For a convolutional code of rate $k / n$ there are $2^{k}$ merging paths at each state in the trellis. The decoding of this code by the Viterbi algorithm selects the path with the highest metric out of the $2^{k}$ possibilities at each state. The number of calculations per selection grows exponentially with $k$, and implementation at high speed is a serious limitation, particularly in the case of high-rate codes. Fortunately, the Viterbi decoding of high-rate codes where $k>1$ can be significantly simplified by employing punctured convolutional codes. ${ }^{19,20}$ This technique allows us to obtain a high-rate code by periodically deleting parts of the coded bits from a low-rate encoder output. In addition, puncturing of the low-rate $1 / n$ code results in the decoding trellis operating with $k$ equal to unity.

We have used the low-rate convolutional code $\operatorname{PCC}(3,1,7)$, punctured to a $2 / 3$ coding rate. This code is appropriate to the inner layer of a concatenated scheme with overall coding rate of $1 / 2$. The generator polynomials of this code are

$$
\begin{aligned}
& g_{0}(D)=1+D+D^{2}+D^{5}+D^{6} \\
& g_{1}(D)=1+D^{2}+D^{3}+D^{4}+D^{6}
\end{aligned}
$$

and

$$
g_{2}(D)=1+D+D^{2}+D^{5}+D^{6}
$$

which are designed to produce an optimum code at $\mathrm{BER}=10^{-5}$ in the presence of an AWGN channel. ${ }^{19}$ The puncturing pattern of the encoded bit sequence is to delete every alternate output bit, starting from the first bit generated by $g_{0}(D)$ and $g_{1}(D)$, and also every alternate output bit, starting from the second bit produced by $g_{2}(D)$.

The weight distribution of this code after puncturing was found by computer search to be

$$
W_{\mathrm{PCC} 317}(d)=d^{6}+44 d^{7}+238 d^{8}+\cdots
$$

from the all-zero path. The post-decoding bit error probability $p_{\mathrm{bp}}$ is found by substituting this weight distribution, and the probability $P_{\mathrm{ICD}}$ of either incorrect decoding using hard decisions given by equation (7) or of using soft decisions given by equation (9) into equation (6).

\section{BER PERFORMANCE OF REED-SOLOMON CODES}

In this section we consider the abilities of Reed-Solomon (RS) codes to combat transmission 


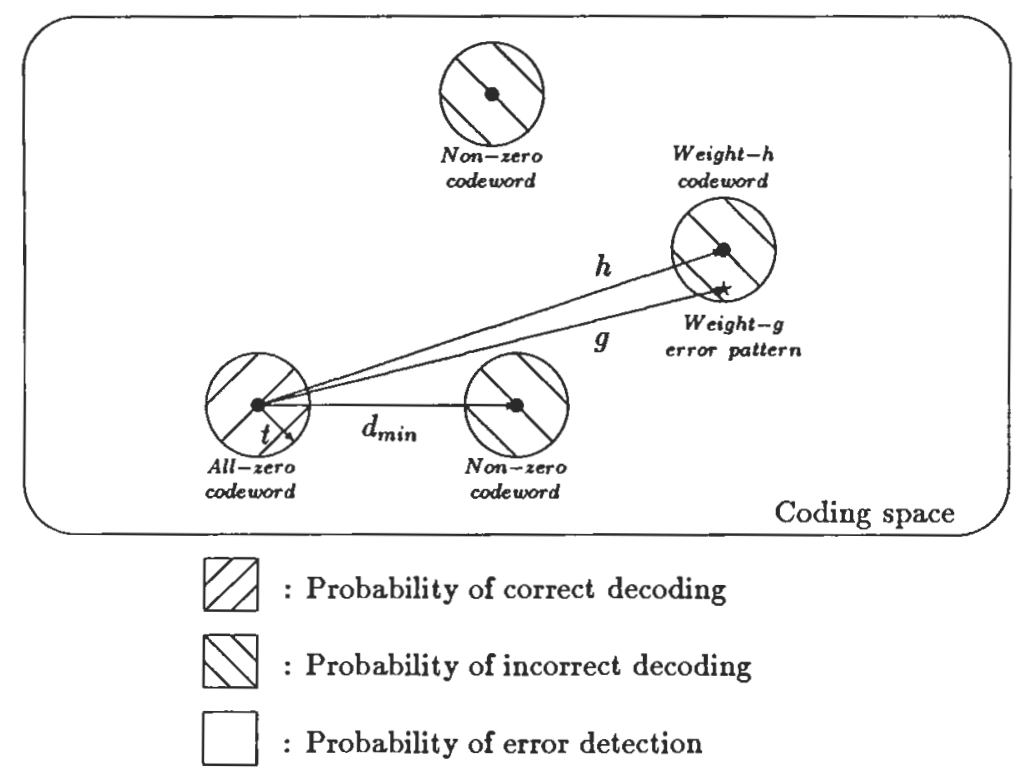

Figure 13. Representation of code words in coding space

errors when the Berlekamp-Massey decoding procedure is employed. The RS code has linear properties. We may therefore analyse its behaviour by considering that a particular code word is transmitted, say the all-zero code word, knowing that our findings are applicable for the transmission of any other code word.

The concept of geometric coding space is particularly useful for visualizing the decoding situation. Figure 13 represents the coding space containing $\left(q^{m}\right)^{n}$ words, of which $\left(q^{m}\right)^{k}$ are code words. If the received word lies within the all-zero code word sphere, it can be corrected. The probability of correct decoding is therefore represented by the ratio of the volume of the all-zero code word sphere to the volume of the total coding space, and the probability of an incorrect decoding is the ratio of the volumes of the code-word spheres to the total volume of the coding space. The probability of error detection is the percentage of the coding space that does not contain coding spheres. Thus we may express the error detection of a code word as

$$
P_{\mathrm{ED}}=1-P_{\mathrm{CD}}-P_{\mathrm{ICD}}
$$

where $P_{\mathrm{CD}}$ and $P_{\mathrm{ICD}}$ are the probabilities of correctly decoding and incorrectly decoding into another valid code word, respectively. Another useful probability is the relative error detection probability

$$
P_{\mathrm{EDR}}=\frac{P_{\mathrm{ED}}}{P_{\mathrm{ED}}+P_{\mathrm{ICD}}}
$$

which is the probability of detecting an uncorrectable error in a code word.

The RS codes considered here are over $\operatorname{GF}\left(q^{m}\right)$, where $q=2$ and $m$ is the number of bits in a symbol. The $m$-bit symbols are transmitted sequentially over a binary channel, which is modelled as an asymmetric memoryless channel, as discussed in Section 3.2. The probability of receiving an error symbol for this channel is given by equation (3), and the probability of receiving a symbol with $i$ bit errors is expressed by equation (2).

\subsection{Probability of correct decoding}

An $\operatorname{RS}(n, k)$ code defined over $\operatorname{GF}\left(q^{m}\right)$ with minimum distance $d_{\min }=n-k+1$ is able to correct $t=(n-k) / 2$ symbol errors. Hence, the probability of correct decoding, $P_{\mathrm{CD}}$ is the probability of receiving an $n$-symbol word having $t$ or fewer symbol errors, and is given by

$$
P_{\mathrm{CD}}=\sum_{i=0}^{\prime}\left(\begin{array}{c}
n \\
i
\end{array}\right)\left[1-\left(1-p_{\mathrm{b}}\right)^{m}\right]^{i}\left[\left(1-p_{\mathrm{b}}\right)^{m}\right]^{n-i}
$$

The index $i$ is the number of error symbols in a code word and ranges from zero to $t$. There are $\left(\begin{array}{c}n \\ i\end{array}\right)$ possible error patterns, and $\left[1-\left(1-p_{\mathrm{b}}\right)^{m}\right]^{i}$ is the probability of $i$ symbols being received in error, while $\left[\left(1-p_{\mathrm{b}}\right)^{m}\right]^{n-i}$ is the probability of $(n-i)$ symbols being received correctly. Notice that for channels having a high $\mathrm{SNR}, p_{\mathrm{b}}$ is very small and $P_{\mathrm{CD}}$ approaches unity.

\subsection{Probability of incorrect decoding}

If the received word contains more than $t$ error symbols, it is either decoded incorrectly, or an error detection flag is raised after identifying uncorrectable 
errors in the received word. The probability of incorrect decoding ${ }^{21}$ is

$$
P_{\mathrm{ICD}}=\sum_{h=d_{\text {min }}}^{n} P_{\mathrm{ICD}}(h)
$$

where $P_{\mathrm{ICD}}(h)$ is the probability of incorrect decoding to a code word having a symbol distance $h$ from the all-zero code word. In order to determine the probability of incorrectly decoding to a weight $h$ code word, we define $N_{g, s}(h)$ as the number of weight-g error patterns that are at a distance $s$ from a particular weight- $h$ code word. When $s \leq t$ and $h \geq d_{\min }$, each such error pattern is decoded incorrectly into the weight- $h$ code word. Suppose $P(g)$ is the probability of occurrence of a particular weight- $g$ error pattern and $A_{h}$ is the number of weight- $h$ code words, then the probability of incorrectly decoding as a weight- $h$ code word is the sum of all the probabilities $P(g)$ of weight- $g$ error patterns which lie inside the decoding sphere of a particular weight- $h$ code word, that is

$$
P_{\mathrm{ICD}}(h)=A_{h} \sum_{s=0}^{l} \sum_{g=h-s}^{h+s} N_{g, s}(h) P(g),
$$

Suppose an all-zero $\operatorname{RS}(n, k)$ code word is transmitted, then the probability of occurrence of single error symbol, which we will refer to as producing a weight- 1 error pattern is

$$
P(1)=\sum_{i_{1}=1}^{m}\left(\begin{array}{c}
m \\
i_{1}
\end{array}\right) p_{b}^{i_{1}}\left(1-p_{\mathrm{b}}\right)^{m n-i_{1}}
$$

The probability of a weight- 2 error pattern is the joint probability of two mutually independent error symbols occurring, and is the product of their individual probabilities, namely

$$
P(2)=\sum_{i_{1}=1 i_{2}=1}^{m} \sum^{m}\left(\begin{array}{c}
m \\
i_{1}
\end{array}\right)\left(\begin{array}{c}
m \\
i_{2}
\end{array}\right) p_{\mathrm{b}}^{i_{1}+i_{2}}\left(1-p_{\mathrm{b}}\right)^{m n-\left(i_{1}+i_{2}\right)}
$$

The probability of receiving a particular weight-1, weight-2, .., error pattern out of the possible $\left(2^{m}-1\right),\left(2^{m}-1\right)^{2}, \ldots$, such patterns is found by dividing $P(1), P(2), \ldots$, by $\left(2^{m}-1\right),\left(2^{m}-1\right)^{2}, \ldots$, and the probability of receiving a particular weight$g$ error pattern is

$$
\begin{aligned}
& P(g)=\frac{1}{\left(2^{m}-1\right)^{g}} \sum_{i_{1}=1}^{m} \sum_{i_{2}=1}^{m} \cdots \sum_{i_{g}=1}^{m} \\
& {\left[\left(\begin{array}{c}
m \\
i_{1}
\end{array}\right)\left(\begin{array}{l}
m \\
i_{2}
\end{array}\right) \cdots\left(\begin{array}{l}
m \\
i_{g}
\end{array}\right)\right.} \\
& \left.p_{d}^{i}+i_{2}+\cdots+i_{g}\left(1-p_{\mathrm{b}}\right)^{m n-\left(i_{1}+i_{2}+\cdots+i_{g}\right)}\right]
\end{aligned}
$$

The number of weight-g error patterns at a distance $s$ from the weight $h$ code word is ${ }^{21}$ expressed by

$$
\begin{aligned}
N_{g, s}(h)= & \sum_{z=z_{\min }}^{z_{\max }}\left(\begin{array}{c}
h \\
h-s+z
\end{array}\right)\left(\begin{array}{c}
s-z \\
g-h+s-2 z
\end{array}\right) \\
& \left(\begin{array}{c}
n-h \\
z
\end{array}\right)\left(2^{m}-2\right)^{g^{-h+s-2 z}\left(2^{m}-1\right)^{z}}
\end{aligned}
$$

where the summation limits $z_{\min }$ and $z_{\max }$ are

$$
z_{\min }=\max \{0, g-h\}
$$

and

$$
z_{\max }=\left[\frac{g-h+s}{2}\right]
$$

where $[ \pm]$ is the truncated integer value of \pm .

To evaluate $P_{\text {ICD }}(h)$ of equation (16) we must compute the weight distribution $A_{h}$. For any maximum distance code such as a Reed-Solomon code defined over GF $\left(q^{m}\right)$ with code-word length $n$ and minimum distance $d$, the weight distribution $A_{h}$ is given by ${ }^{12}$

$A_{h}=\left(\begin{array}{l}n \\ h\end{array}\right)\left(q^{m}-1\right) \sum_{j=0}^{h-d}(-1)^{j}\left(\begin{array}{c}h-1 \\ j\end{array}\right)\left(q^{m}\right)^{h-d-j}$

By substituting $P(g), N_{g, s}(h)$ and $A_{h}$ from equations $(19,(20)$ and (23) into equation (16) and then substituting $P_{\mathrm{ICD}}(h)$ into equation (15), we have

$$
\begin{aligned}
& P_{\mathrm{ICD}}=\sum_{h=d}^{n}\left[\left(\begin{array}{l}
n \\
h
\end{array}\right)\left(q^{m}-1\right) \sum_{j=0}^{h-d}(-1)^{j}\right. \\
& \left.\left(\begin{array}{c}
h-1 \\
j
\end{array}\right)\left(q^{m t}\right)^{h-d-j}\right] \sum_{s=0} \sum_{g=h-s}^{h+s}\left\{\left[\sum_{z=z_{\min }}^{z_{\max }}\right.\right. \\
& \left(\begin{array}{c}
h \\
h-s+z
\end{array}\right)\left(\begin{array}{c}
s-z \\
g-h+s-2 z
\end{array}\right) \\
& \left.\left(\begin{array}{c}
n-h \\
z
\end{array}\right)\left(2^{m}-2\right)^{g^{-h+s-2 z}}\left(2^{m}-1\right)^{z}\right] \\
& {\left[\frac { 1 } { ( 2 ^ { m } - 1 ) ^ { g } } \sum _ { i _ { 1 } = 1 } ^ { m } \sum _ { i _ { 2 } = 1 } ^ { m } \ldots \sum _ { i _ { g } = 1 } ^ { m } \left[\left(\begin{array}{c}
m \\
i_{1}
\end{array}\right)\left(\begin{array}{c}
m \\
i_{2}
\end{array}\right) \ldots\left(\begin{array}{c}
m \\
i_{g}
\end{array}\right)\right.\right.} \\
& \left.\left.\left.\mathrm{p}_{\mathrm{h}}{ }^{i_{1}+i_{2}+\cdots+i_{g}}\left(1-p_{\mathrm{h}}\right)^{m m-\left(i_{1}+i_{2}+\cdots+i_{g}\right)}\right]\right]\right\}
\end{aligned}
$$

Observe that $P_{\mathrm{ICD}}$ tends to zero if the SNR is high, as $p_{\mathrm{b}}$ is approximately zero. Armed with $P_{\mathrm{CD}}$ and $P_{\mathrm{ICD}}$ the probability of error detection $P_{\mathrm{ED}}$ is computed using equation (12), whereas the relative error detection probability, $P_{\mathrm{EDR}}$ is determined using equation (13). 


\subsection{Post-decoding bit and symbol error probabilities}

When the received code word contains more than t symbol errors they are either known to be incorrect, or they are decoded into another code word and the error is unknown. We will now determine the probability of the symbols and the bits in the regenerated code word being in error for the cases when the errors are known and unknown.

When errors are detected a systematic RS code conveys the information part of the code word directly to the decoder output as decoded information. Consequently, the post-decoding error probability is equal to the pre-decoding error probability. Hence the contributions to the respective bit and symbol error probabilities $p_{\mathrm{bpl}}$ and $p_{\mathrm{spl}}$ are

$$
\begin{aligned}
p_{\mathrm{bp} 1} & =p_{\mathrm{b}} P_{\mathrm{ED}} \\
p_{\mathrm{sp} 1} & =\sum_{i=1}^{m}\left(\begin{array}{c}
m \\
i
\end{array}\right) p_{\mathrm{bp} 1}^{i}\left(1-p_{\mathrm{hp} 1}\right)^{m-i}
\end{aligned}
$$

For error patterns that are undetectable the received word is decoded as a weight- $h$ code word, i.e. it contains $h$ error symbols. The post-decoding symbol error probability $p_{\mathrm{sp} 2}$ is given by

$$
p_{\mathrm{sp} 2}=\frac{1}{n} \sum_{h=d}^{n} h P_{\mathrm{ICD}}(h)
$$

where $P_{\mathrm{ICD}}(h)$ is given by equation (16). The postdecoding bit error probability due to incorrect decoding can be evaluated from $p_{\mathrm{sp} 2}$ as follows:

$$
\begin{aligned}
& p_{\mathrm{sp} 2}=1-\left(1-p_{\mathrm{hp} 2}\right)^{m} \\
& p_{\mathrm{hp} 2}=1-\mathrm{e}^{\frac{1}{m} \ln \left(1-p_{\mathrm{sp} 2}\right)}
\end{aligned}
$$

The total post-decoding symbol error probability $p_{s p}$ and the total post-decoding bit error probability $p_{\mathrm{bp}}$ can be expressed in terms of the probability of predecoding bit errors $p_{\mathrm{b}}$, the probability of error detection $P_{\mathrm{ED}}$ and the probability of incorrect decoding $P_{\mathrm{ICD}}$, namely

$$
\begin{aligned}
p_{\mathrm{sp}} & =p_{\mathrm{sp} 1}+p_{\mathrm{sp} 2} \\
& =\sum_{i=1}^{m}\left[\left(\begin{array}{c}
m \\
i
\end{array}\right)\left(p_{\mathrm{b}} P_{\mathrm{ED}}\right)^{i}\left(1-p_{\mathrm{b}} P_{\mathrm{ED}}\right)^{m-i}\right] \\
& +\frac{1}{n} \sum_{l i=d}^{n} h P_{\mathrm{lCD}}(h)
\end{aligned}
$$

and

$$
\begin{aligned}
p_{\mathrm{hp}} & =p_{\mathrm{hp} 1}+p_{\mathrm{hp} 2} \\
& =p_{\mathrm{b}} P_{\mathrm{ED}}+\left[1-\mathrm{e}^{\frac{1}{m} \ln \left(1-\frac{1}{n}{ }_{h} \stackrel{n=}{n=d} h P_{\mathrm{ICD}}(h)\right)}\right]
\end{aligned}
$$

\section{TRELLIS DECODING OF BLOCK CODES}

The method of trellis decoding of block codes is similar to Viterbi decoding of convolutional codes. The block decoder selects the path in the trellis having the smallest distance from the received word as the recovered code word. The distance properties of the code determine its error-correcting capability. In Section 6.1 and 6.2, we determine the code-word distances of binary and non-binary block codes with the same decoding complexity, i.e. with the same number of states in the trellis. The codes with this complexity equivalence, having 256 states, are the $\mathrm{BCH}(15,7)$ code and the $\mathrm{RS}(4,2), \mathrm{GF}(16)$ code. In the following subsections their BER performances are evaluated.

\subsection{The $\mathrm{BCH}(15,7)$ code}

The distance properties of this binary block code are derived in a similar way to those of the convolutional code, which suggest that this code can correct any combination of two-bit errors. For convolutional codes the number of incorrect paths increases exponentially and indefinitely with the number of columns $j$ in the trellis. However, block codes have a fixed code-word length, and consequently the trellis is truncated after $n$ columns. The paths in the trellis initially diverge from, and finally converge to, the all-zero state at the codeword boundaries. All the paths have the same length of $n$ bits, there is a total number of $2^{k}$ possible paths in the trellis and the weight distribution, $W_{\mathrm{BCH} 157}(d)$, of the $\mathrm{BCH}(15,7)$ code was found by computer search through all of these paths as

$$
\begin{aligned}
W_{\mathrm{BCH} 1.57}(d) & =42 d^{5}+84 d^{6}+49 d^{7}+56 d^{8} \\
& +126 d^{9}+84 d^{10}+7 d^{15}
\end{aligned}
$$

The minimum separable distance, $d_{\text {min }}$, between the code words is 5 bits. We will represent the total number of information bit errors for all those paths having a distance $d$ by the coefficient $W_{d}$. Following the procedure of Section 4.1, the union bound on the post-decoding bit error probability $p_{\mathrm{bp}}$ for binary block codes is

$$
p_{\mathrm{bp}} \leq \sum_{d=\iota_{\mathrm{tmin}}}^{n} W_{d} P_{\mathrm{ICD}}(d)
$$

where $P_{\mathrm{ICD}}(d)$ is the probability of incorrect decoding, i.e. the probability that the decoder selects a path at distance $d$ from the correct path. The value of $P_{\mathrm{ICD}}(d)$ is found from equation (7) for harddecision decoding, or from equation (9) for softdecision decoding.

\subsection{The $\mathrm{RS}(4,2)$ code}

Reed-Solomon codes have a maximum separable distance between code words of $(n-k+1)$ symbols. 


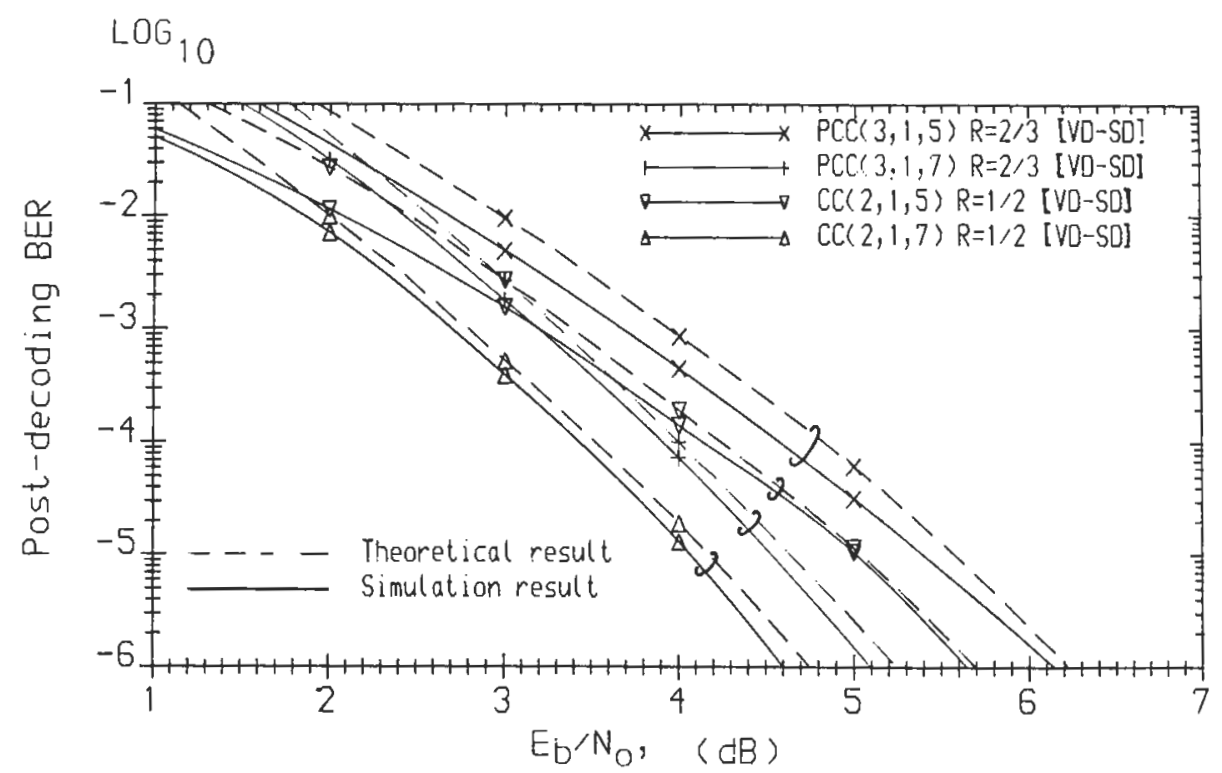

Figure 14. Post-decoding BER of CC decoded by using the Viterbi algorithm with soft-decision decoding over a Gaussian channel

Optimum symbol-by-symbol decoding methods 22.23 that minimize the symbol error rate result in increased complexity compared to Viterbi decoding, while the performance is essentially the same. Consequently, we decode using a bit-orientated Viterbi algorithm and describe the properties of the RS code in terms of bit-distance measures. For the RS(4,2), GF(16) code the weight distribution $W_{\mathrm{RS} 42}(d)$ is found by computer search to be

$$
\begin{aligned}
W_{\mathrm{RS} 42}(d) & =14 d^{4}+58 d^{5}+86 d^{6} \\
& +134 d^{7}+210 d^{8}+218 d^{9}+170 d^{10} \\
& +82 d^{11}+24 d^{12}+20 d^{13}+8 d^{14}
\end{aligned}
$$

The union bound on the post-decoding bit error probability $p_{\text {bp }}$ for this code is obtained by substituting $W_{\mathrm{RS} 42}(d)$ into equation (33).

\section{RESULTS FOR NON-CONCATENATED CODES}

In Figure 14 we display a set of theoretical and simulation results for the convolutional codes $\mathrm{CC}(2,1,7), \mathrm{CC}(2,1,5)$ and the punctured 2/3-rate convolutional codes $\operatorname{PCC}(3,1,7)$ and $\operatorname{PCC}(3,1,5)$. All codes are decoded by soft-decision Viterbi decoding. The transmissions were via MSK over an AWGN channel. The theoretical and simulation curves are seen to be in close agreement. The 2/3-rate punctured code with constraint length 7 has a $0.5 \mathrm{~dB}$ coding gain in $E_{\mathrm{b}} / N_{0}$ at a BER of $10^{-5}$ compared to the $1 / 2$-rate code of constraint length 5 . Thus the lower transmitted bit rate, but greater complexity 2/3-rate decoder has a better performance than that of the 1/2-rate code which transmits at a higher bit rate.
When the transmissions were over Rayleigh fading channels we obtained the results shown in Figure 15 for the $\operatorname{CC}(2,1,7)$ 1/2-rate code and those displayed in Figure 16 for the $\operatorname{PCC}(3,1,7)$ punctured 2/3-rate code. The effect of interleaving with a period of 4032 bits compared to 2880 bits yielded a gain in $E_{\mathrm{b}} / N_{0}$ of only about $0.5 \mathrm{~dB}$. Consequently the interleaving distance of 2880 bits was preferred. The PCC $(3,1,7)$ required a higher channel SNR for a given $p_{\mathrm{bp}}$, as shown in Figure 16, than the other codes, but because of its lower bit rate and adequate BER performance it was elected as the inner code in our concatenated coding scheme.

We now compare the theoretical and simulation results for a number of Reed-Solomon codes having different code-word lengths operating at coding rate $1 / 2$. The Berlekamp-Massey hard-decision decoding method was used. Figure 17 shows the probability of correct decoding as a function of $E_{\mathrm{b}} / N_{\mathrm{0}}$ for the AWGN channel. The probability of correct decoding $P_{\mathrm{CD}}$ using shorter RS codes was found to be higher than for the longer codes for $E_{\mathrm{b}} / N_{0}$ values below approximately $6 \mathrm{~dB}$. This was because the symbol error probability for codes with larger numbers of bits per symbol was higher, as exemplified by Figure 11. This situation was reversed for higher $E_{\mathrm{b}} / N_{0}$ values because longer codes generally have a higher error correcting capability. The simulation and theoretical results coincide.

The probability of incorrect decoding, $P_{\mathrm{ICD}}$ is significantly lower for longer codes because the minimum distance among code words is much larger in the longer codes. Figure 18 shows $P_{\mathrm{ICD}}$ as a function of $E_{1} / N_{0}$ for $\operatorname{RS}(4,2)$ and $\operatorname{RS}(12,6)$ codes. Although not displayed in Figure 18, the theoretical result shows that $P_{\mathrm{ICD}}$ of the $\mathrm{RS}(57,29)$ code is $10^{-23}$ at an $E_{\mathrm{b}} / N_{0}$ of $3 \mathrm{~dB}$. However, the corresponding $P_{\mathrm{ICD}}$ for the short $\mathrm{RS}(4,2)$ and $\mathrm{RS}(12,6)$ codes 


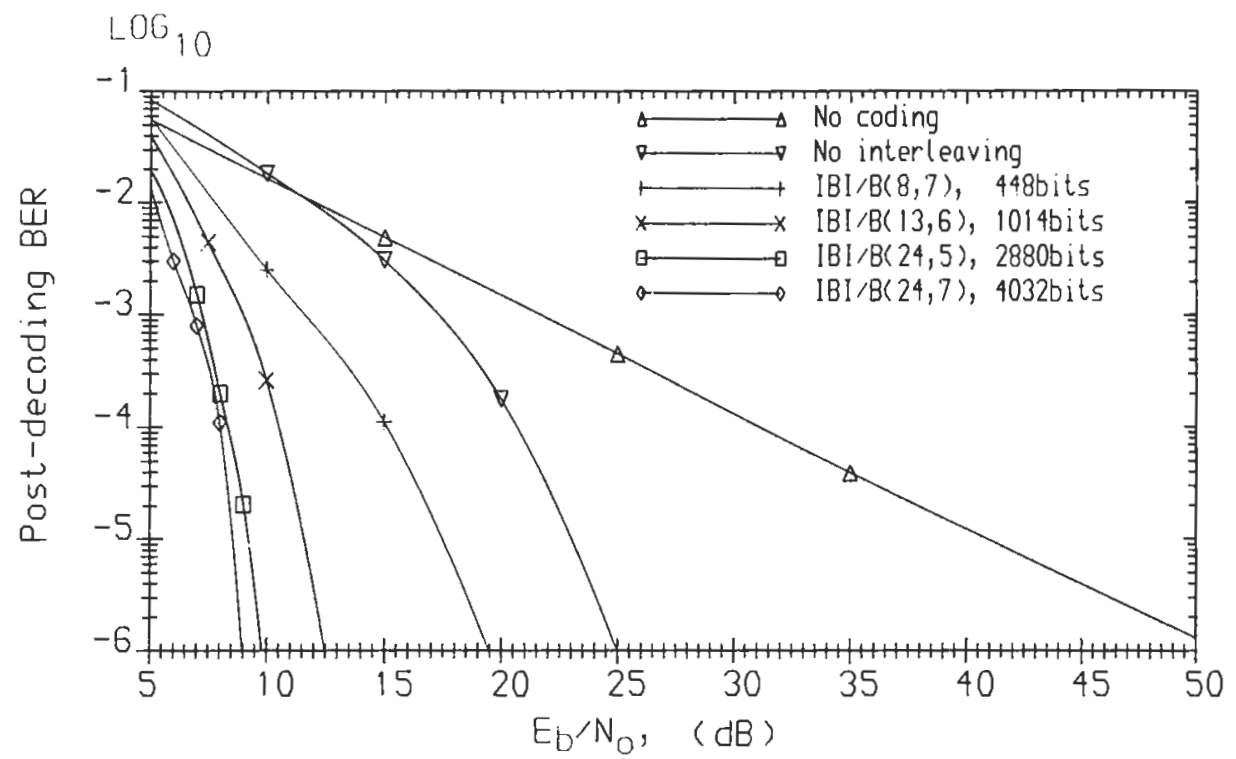

Figure 15. Effect of inter-block interleaving on post-decoding $\mathrm{BER}$ of $\mathrm{CC}(2,1,7), R=1 / 2$ [VD-SD] over a Rayleigh fading channel

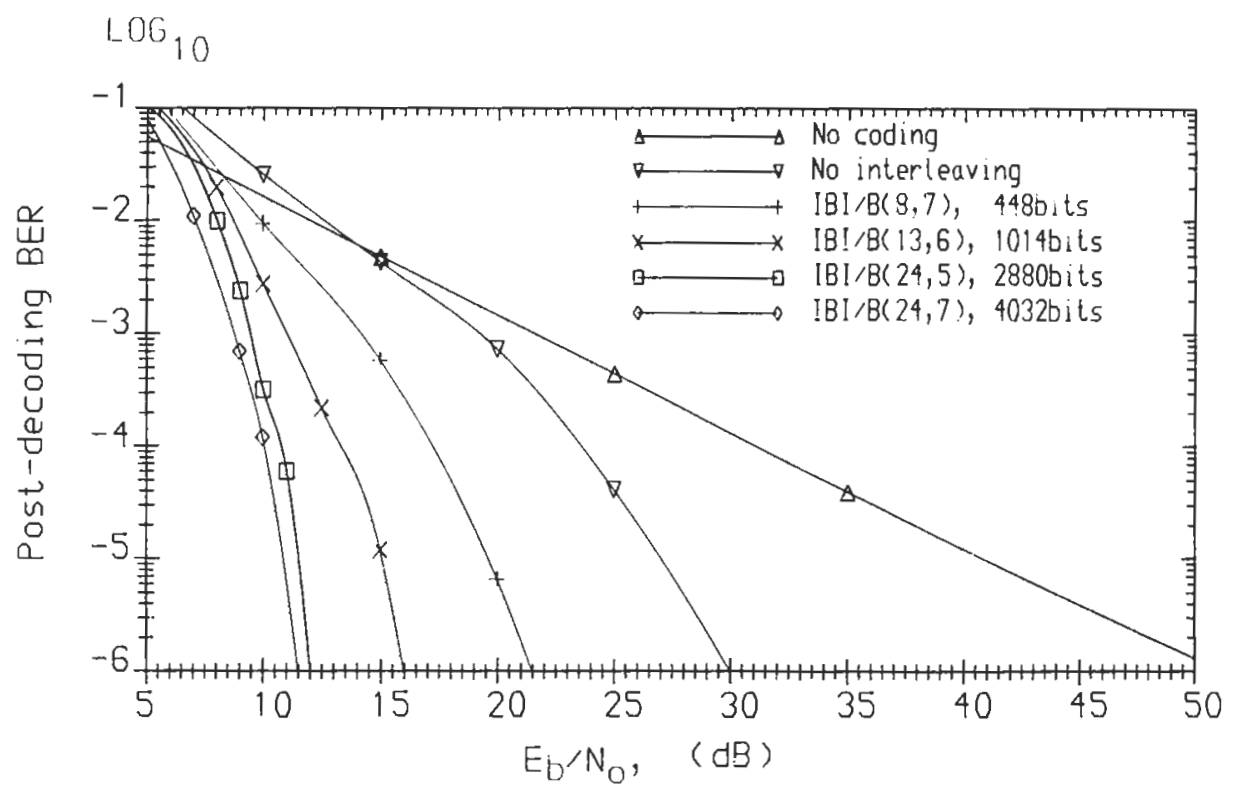

Figure 16. Effect of inter-block interleaving on post-decoding $\mathrm{BER}$ of $\mathrm{PCC}(3,1,7), R=2 / 3$ [VD-SD] over a Rayleigh fading channel

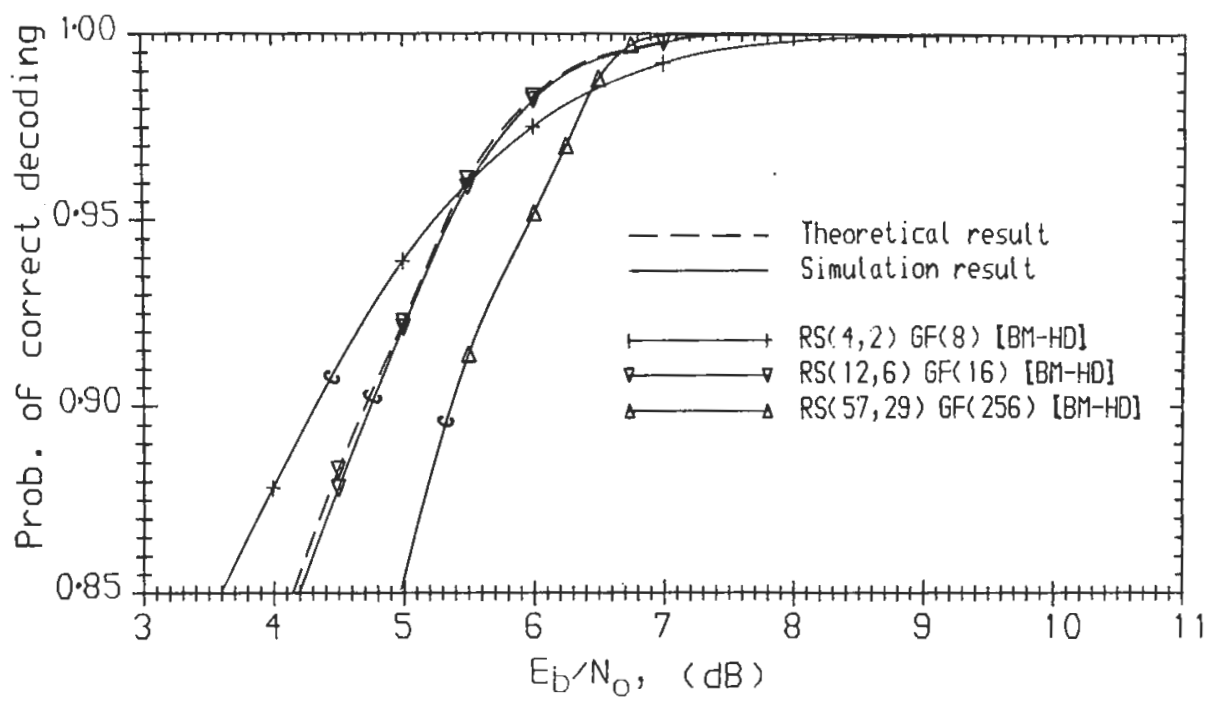

Figure 17. Probability of correct decoding of RS codes over a Gaussian channel 


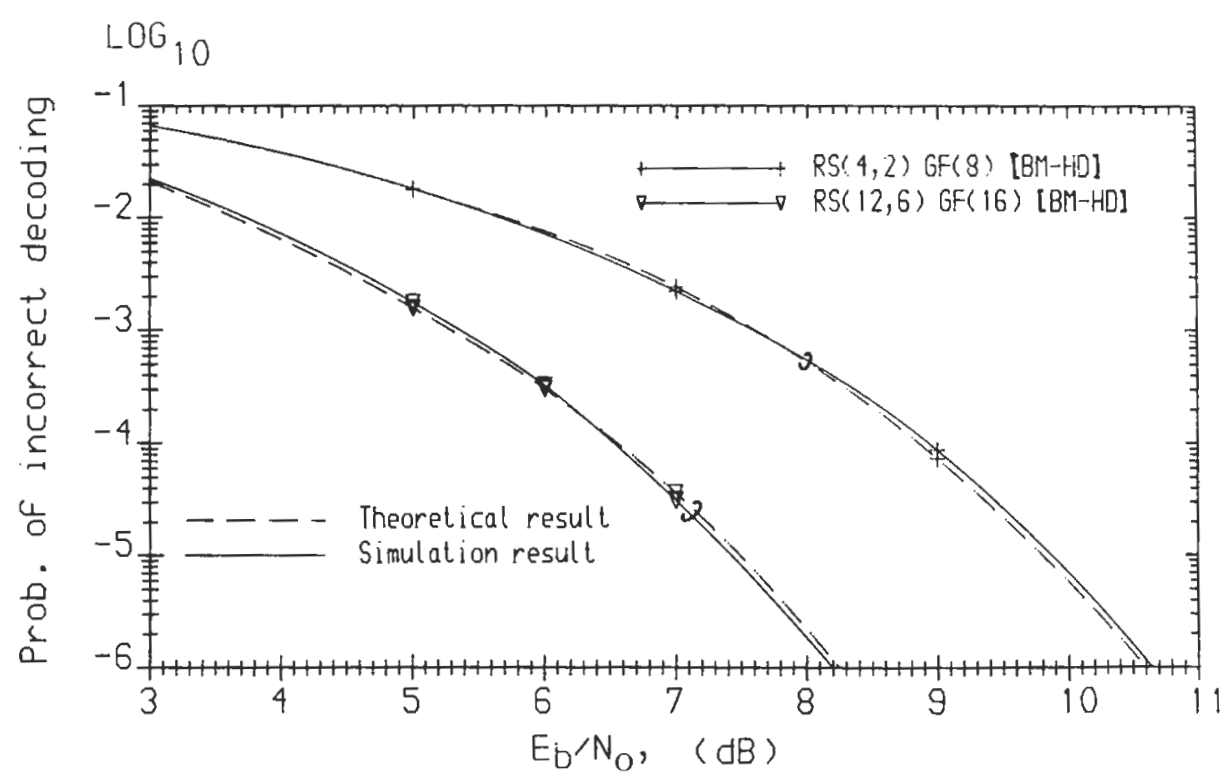

Figure 18. Probability of incorrect decoding of RS codes over a Gaussian channel

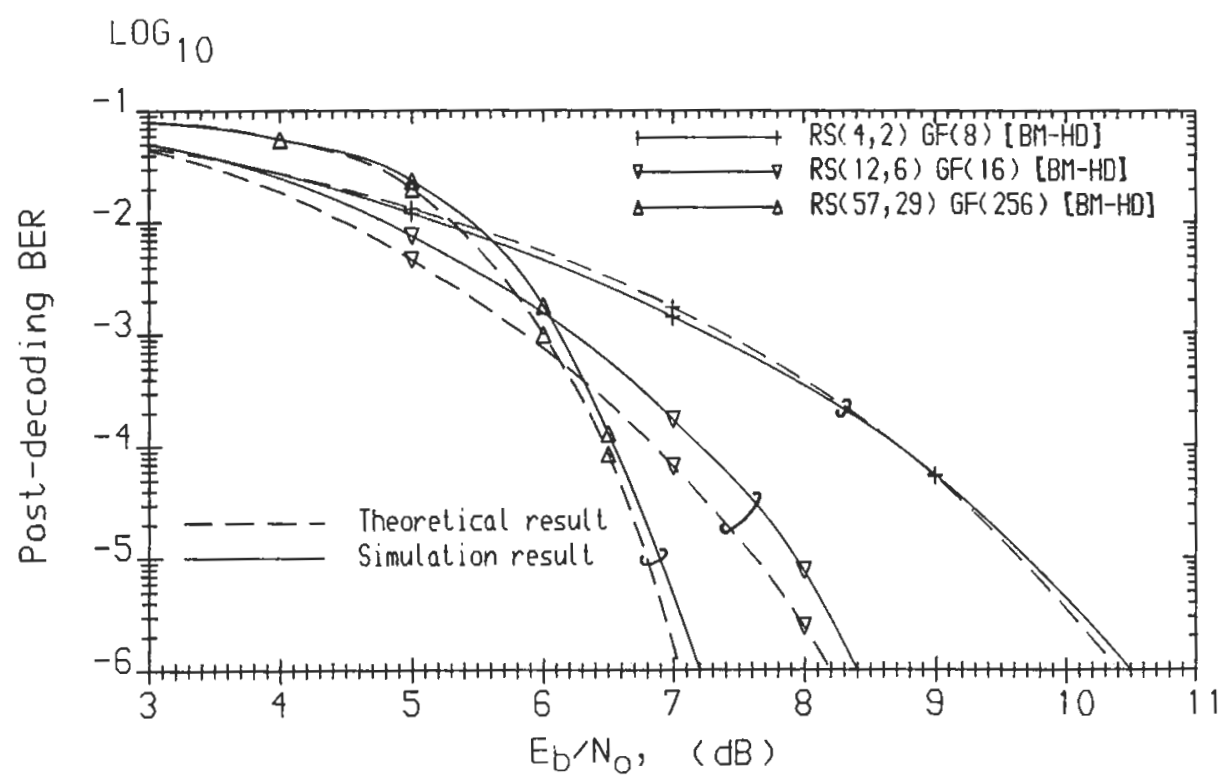

Figure 19. Post-decoding BER of RS codes over a Gaussian channel

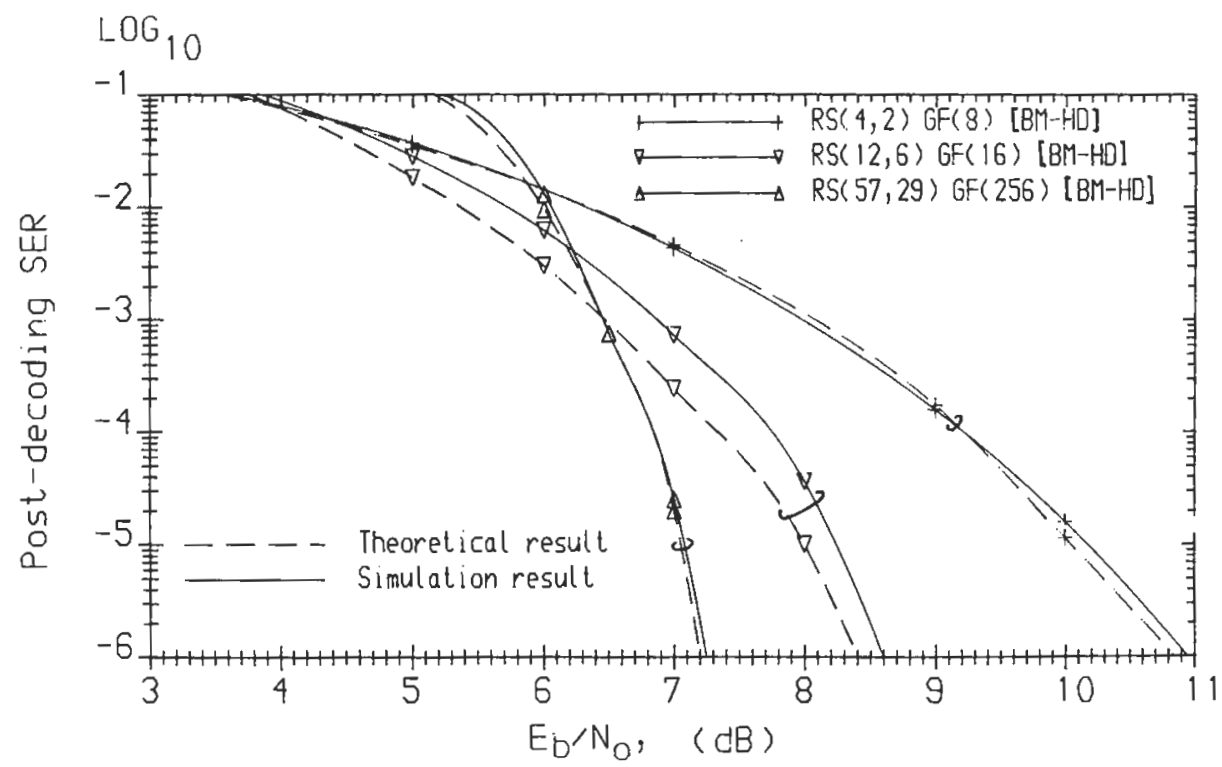

Figure 20. Post-decoding SER of RS codes over a Gaussian channel 


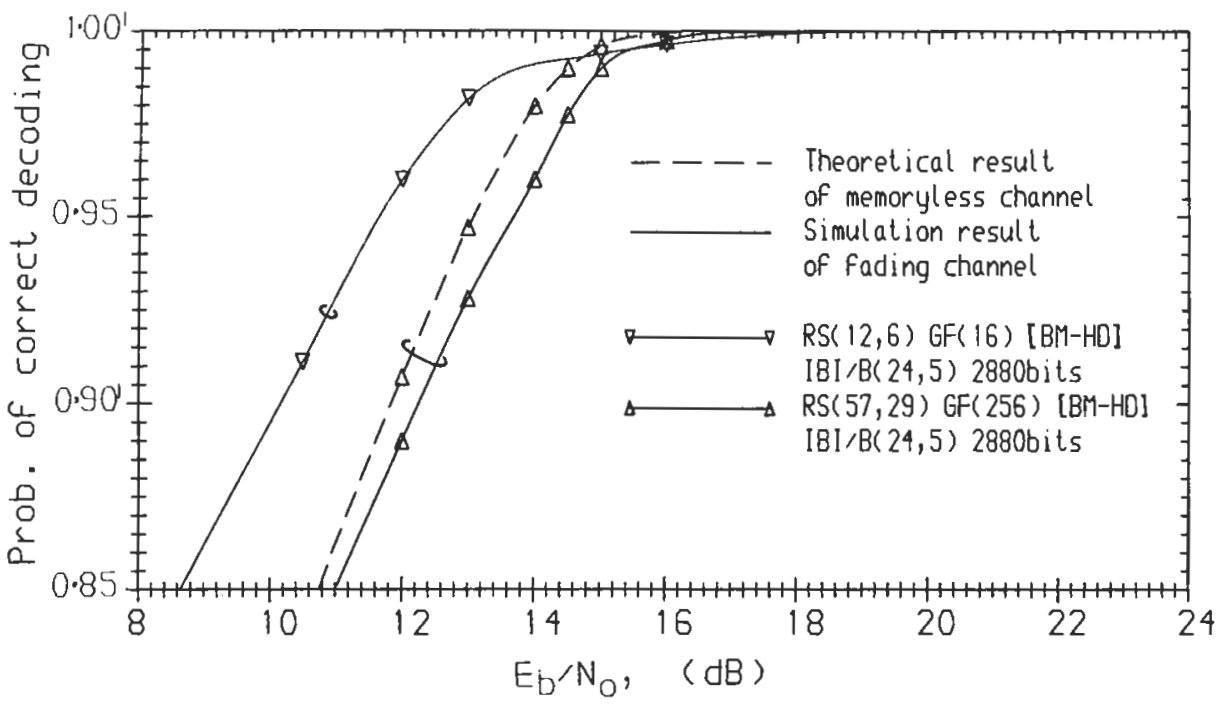

Figure 21. Probability of correct decoding of RS codes over a Rayleigh fading channel

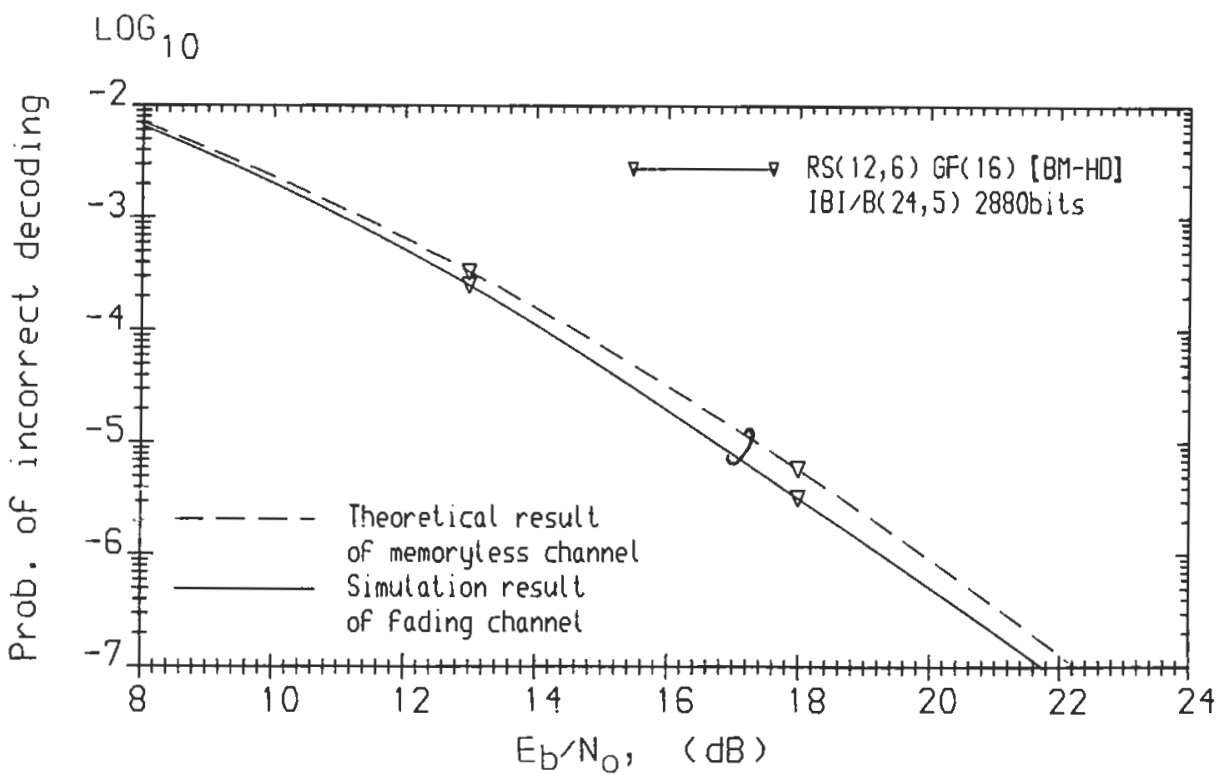

Figure 22. Probability of incorrect decoding of RS codes over a Rayleigh fading channel

are the much higher values of $6.5 \times 10^{-2}$ and 1.2 $\times 10^{-2}$, respectively. Again the simulation and theoretical values coincide.

A very important feature of RS codes is their capability to detect uncorrectable error patterns, the probability of which is characterized in terms of the relative error detecting probability $P_{\text {EDR }}$ as defined in equation (13). As we have experienced, $P_{\mathrm{EDR}}$ is practically independent of $E_{\mathrm{b}} / N_{0}$ for a particular code. For the short RS $(4,2)$ code, the average value of $P_{\text {EDR }}$ is 0.73 , for the $\operatorname{RS}(12,6)$ code it is 0.98 , whereas for the longest code the average $P_{\mathrm{EDR}}$ is $1-10^{-23}$. This suggests that the longest code offers almost certain error detection, an important feature when transmitting computer data.

The post-decoding bit error probability $p_{\mathrm{bp}}$ is a function of both $P_{\mathrm{ICD}}$ and $P_{\mathrm{ED}}$ (see equation (31)). The simulation and theoretical results for a Gaussian channel are displayed in Figure 19 for the three codes. The $p_{\text {bp }}$ curves exhibit again a cross-over region for $E_{\mathrm{b}} / N_{0}$ of between $5 \mathrm{~dB}$ and $6 \mathrm{~dB}$. Below this region the bit error rate of the $\mathrm{RS}(57,29)$ code is the highest as it has a higher channel symbol error rate than the other two codes, as shown in Figure 11. For $E_{\mathrm{b}} / N_{0}$ in excess of $6 \mathrm{~dB}$ the $\operatorname{RS}(57,29)$ code has the highest $p_{\mathrm{bp}}$ performance as the noise corrupting the transmitted code word is restricted for most of the time to the confines of the decoding sphere of that code word. The same tendency can be observed in Figure 20, where the post-decoding symbol error probability $p_{\mathrm{sp}}$ is displayed for the same conditions as those in Figure 19.

The performance of $\operatorname{RS}(12,6)$ and $\operatorname{RS}(57,29)$ codes for MSK transmissions over Rayleigh fading channels is considered next. In Figure 21, we display the probability of correctly decoding a code word as a function of $E_{\mathrm{b}} / N_{0}$. For $E_{\mathrm{b}} / N_{0}=11 \mathrm{~dB}$, the probability of correctly decoding $P_{\mathrm{CD}}$ was approximately 0.86 for the $\operatorname{RS}(57,29)$ code and 0.92 for the RS $(12,6)$ code. The longer code performs better than the shorter code over almost the whole range of $E_{\mathrm{b}} / N_{0}$. When bit or symbol interleaving 


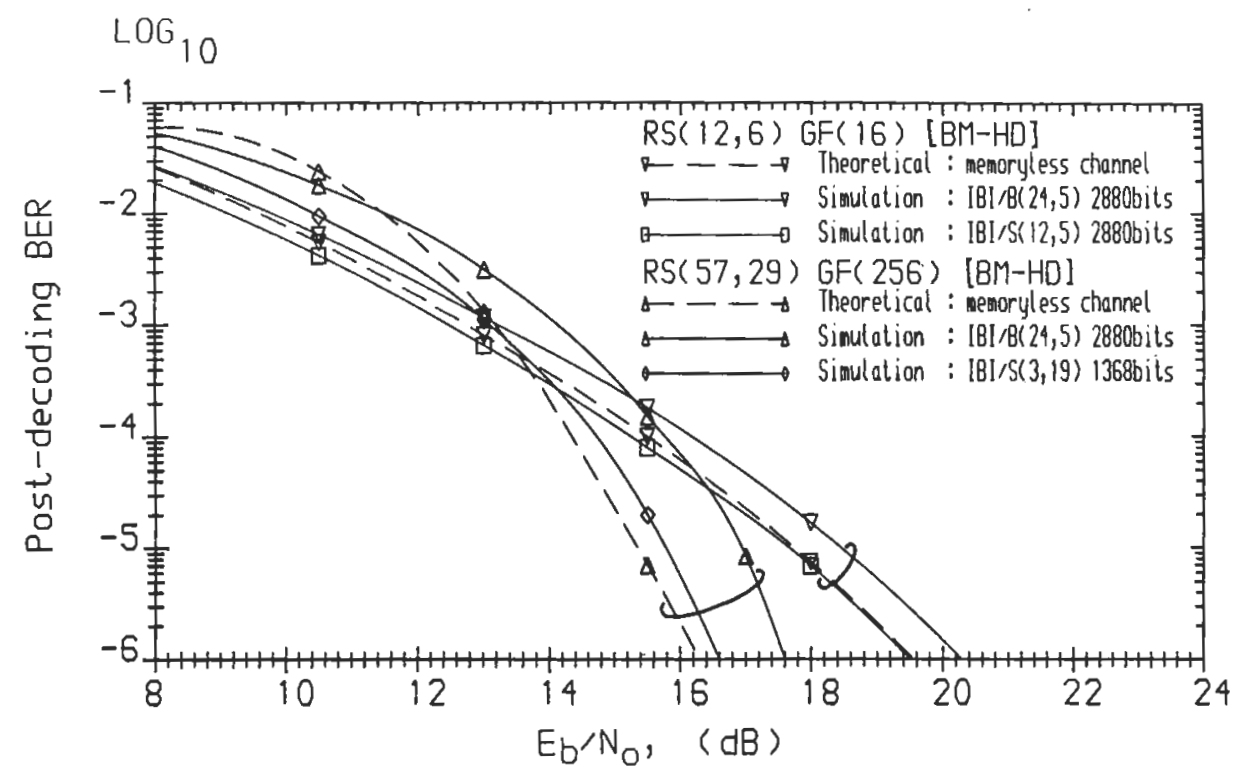

Figure 23. Post-decoding BER of RS codes over a Rayleigh fading channel

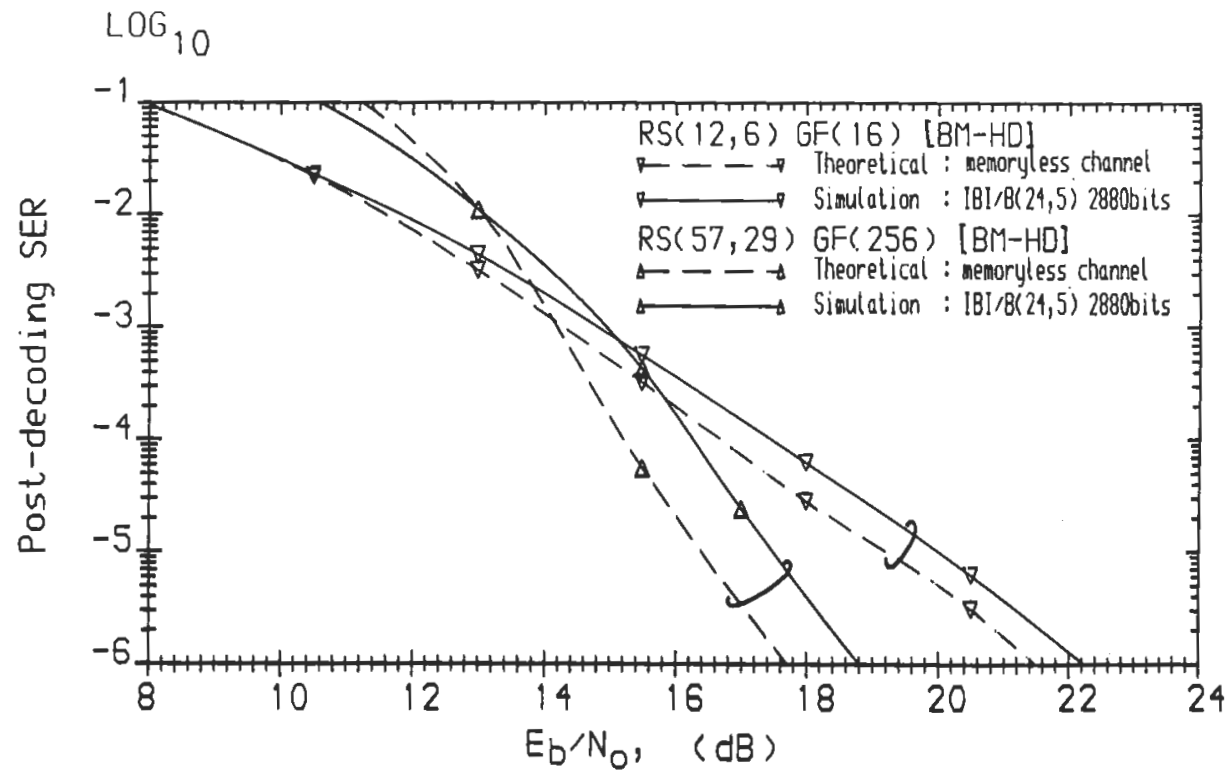

Figure 24. Post-decoding SER of RS codes over a Rayleigh fading channel

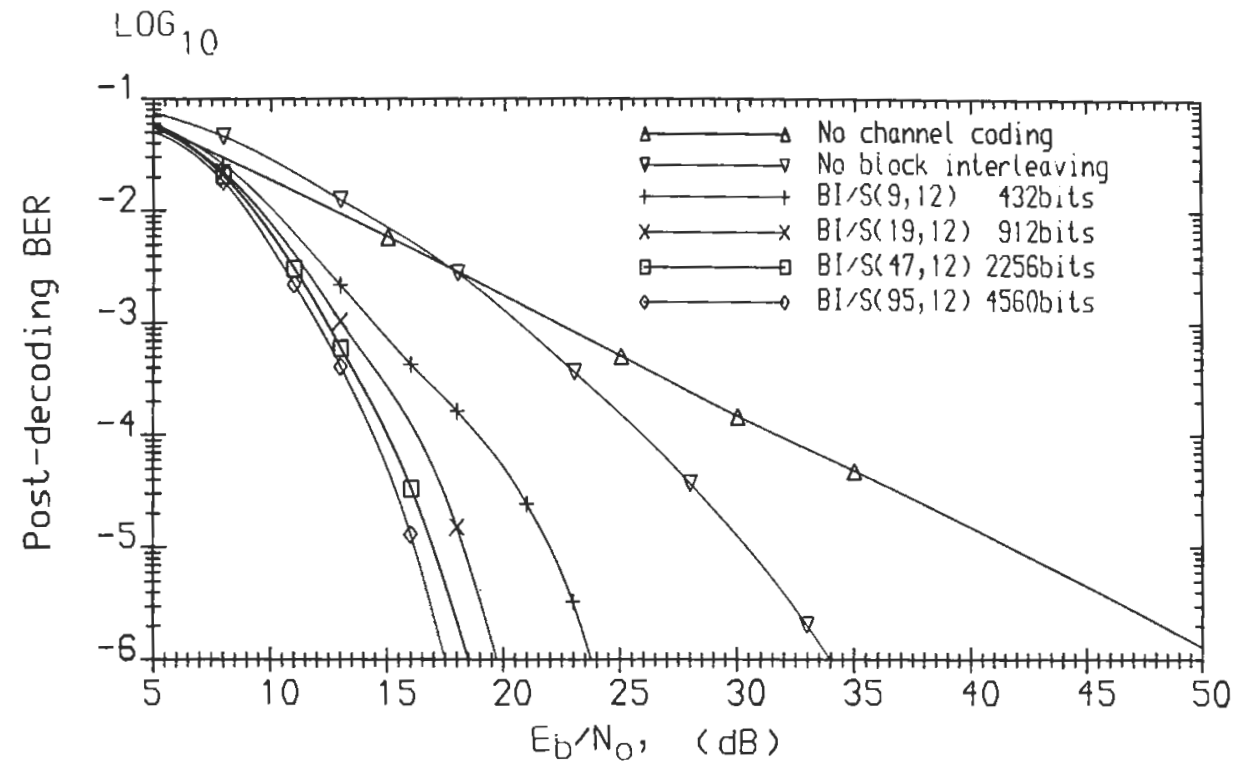

Figure 25. Effect of block interleaving on post-decoding BER of RS(12,6) GF(16) [BM-HD] over a Rayleigh fading channel 


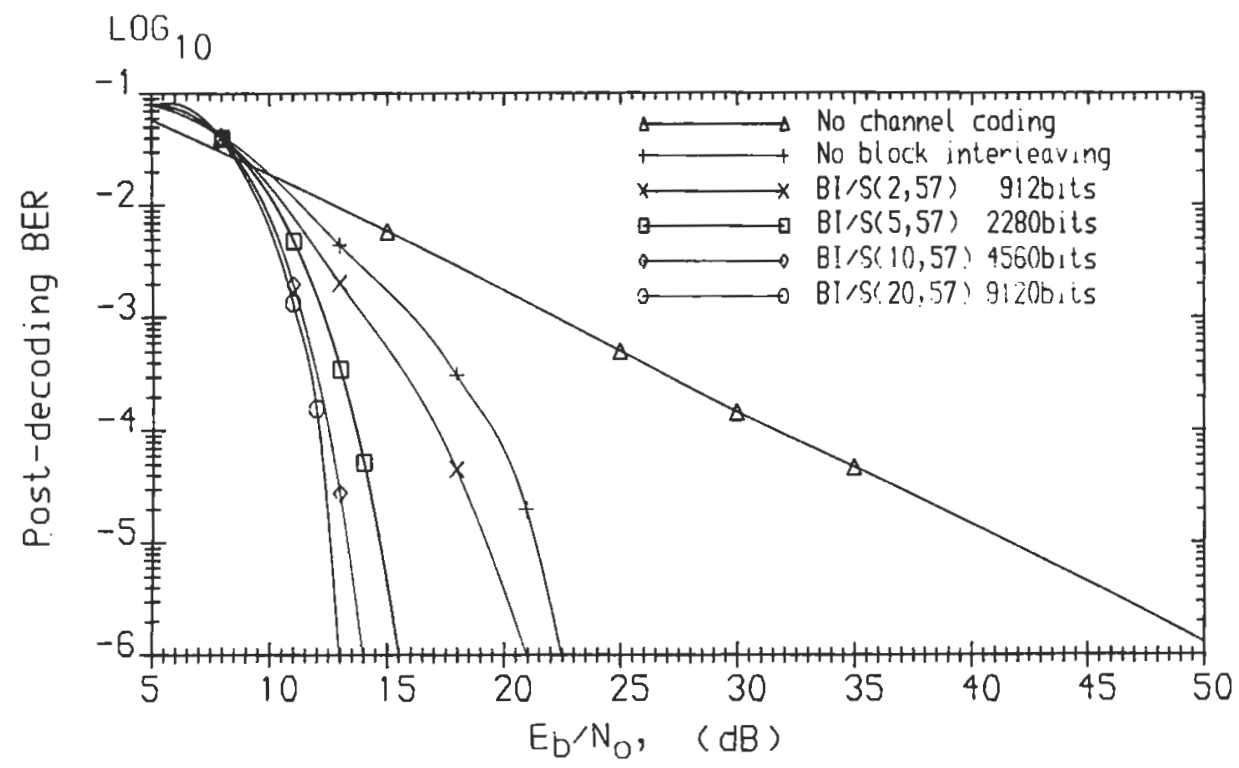

Figure 26. Effect of block interleaving on post-decoding BER of RS(57,29) GF(256) [BM-HD] over a Rayleigh fading channel

was deployed the $P_{\mathrm{CD}}$ was increased for both codes, and the longer the interleaving period the better was the performance. However, a bit-interleaving period of 2880 bits appears to be sufficient to randomize the error statistics of the channel, and results in a performance very similar to the theoretical values. When symbol interleaving was used an interleaving period of only 1368 bits yielded a comparable performance at a considerably lower system delay. This is in harmony with our explanations in Section 3, where we show that symbol interleaving guarantees a better performance for RS codes than bit interleaving.

The probability of incorrect decoding, $P_{\mathrm{ICD}}$, over Rayleigh fading channels is displayed in Figure 22 for the RS $(12,6)$ code with inter-block bit interleaving having a period of 2880 bits. Also the theoretical $P_{\mathrm{ICD}}$ curve is depicted, to show the performance in the case of infinitely long interleaving period, which characterizes the performance over the memoryless channel. For the RS $(57,29)$ code the $P_{\mathrm{ICD}}$ was less than $10^{-23}$ when $E_{\mathrm{b}} / N_{0}$ exceeded $10 \cdot 5 \mathrm{~dB}$, and therefore its performance curve cannot be shown in Figure 22.

The BER performances of the $\mathrm{RS}(12,6)$ and RS $(57,29)$ codes are compared in Figure 23 in terms of their post-decoding bit error probabilities with 2880 bits inter-block interleaving, as well as with two different symbol interleaving periods. First, we focus our attention on the RS $(12,6)$ code, where we observe that even if the bit interleaving period is 2880 bits long, the BER performance is worse than the theoretical bound, computed for the memoryless channel. However, if symbol interleaving is used over the same period via the Rayleigh fading channel, a better BER performance is achieved than that over the memoryless channel. The same tendency is noted in the case of the longer RS $(57,29)$ code, where even a shorter symbol interleaving period of 1368 bits resulted in higher performance than that of the memoryless channel. The BER curves of the two codes have a cross-over zone around $10 \mathrm{~dB}$, and above this value the longer code performs consistently better. In summary, for non-binary $\mathrm{RS}$ codes, symbol interleaving has a more favourable effect than bit interleaving. This experience is confirmed in Figure 24 also in terms of symbol error rates (SER).

In Figures 25 and 26 we explore the performances of the RS $(12,6)$ as well as the $\operatorname{RS}(57,29)$ code over the Rayleigh fading channel in the case of various interleaving periods, when rectangular block symbol interleaving is used. Observe that the shorter RS $(12,6)$ code reaches its saturation at around an interleaving delay of 912 bits, whereas the longer RS $(57,29)$ saturates at around a delay of 2280 bits. However, this longer interleaving period results in a superior BER performance when compared to the shorter code.

In Figure 27 the bit error probabilities of the $\mathrm{RS}(4,2)$ code over $\mathrm{GF}(16)$ and of the $\mathrm{BCH}(15,7)$ code are depicted both with hard-decision decoding and soft-decision trellis decoding over the AWGN channel. In the case of both soft and hard decisions the $\mathrm{BCH}(15,7)$ code performs better than the RS $(4,2)$ code, because it can correct any combination of two bit errors, whereas the RS code copes only with those cases when they occur in the same fourbit symbol, since it can correct only one symbol error per four-symbol code word.

\section{RESULTS FOR CONCATENATED CODES}

In Sections 2-6, we investigated the properties of interleavers, convolutional codes and block codes as separate entities. We now return to the complete system block diagram shown in Figure 1, and consider the assignment of channel coders to the 


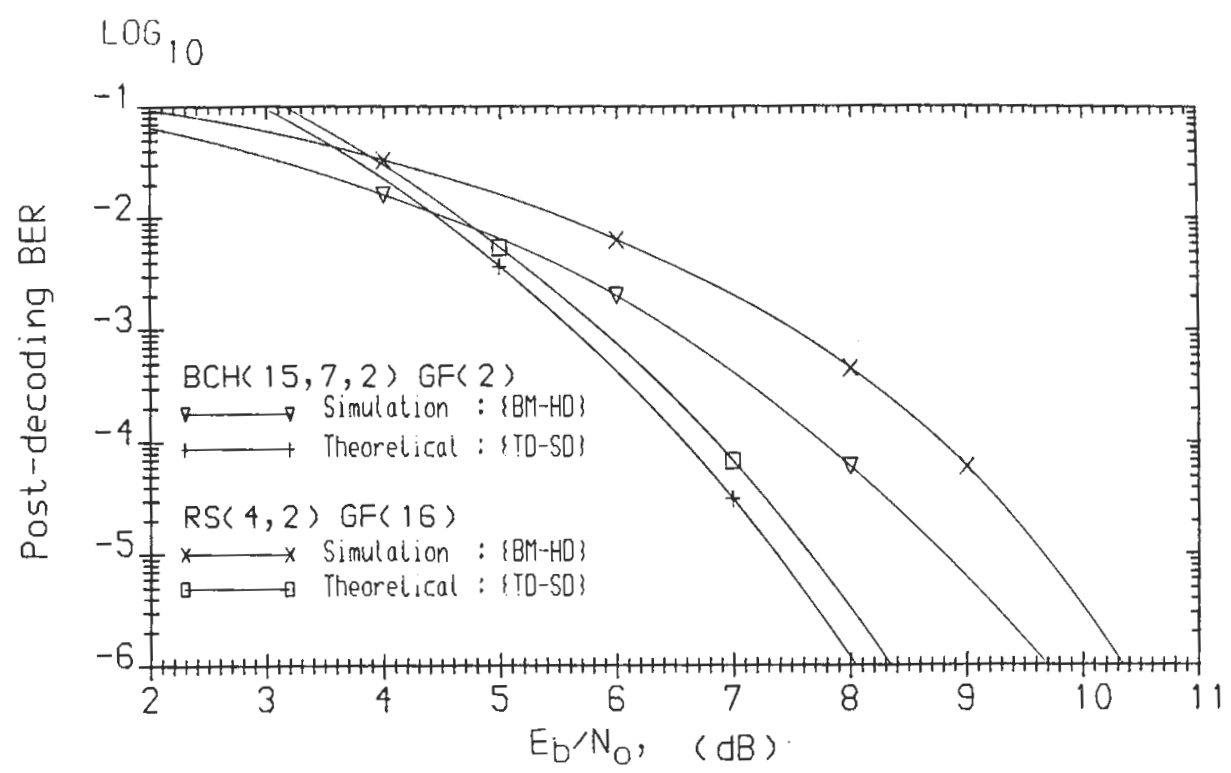

Figure 27. Effect of hard/soft decision on post-decoding BER of Block codes over Gaussian channel

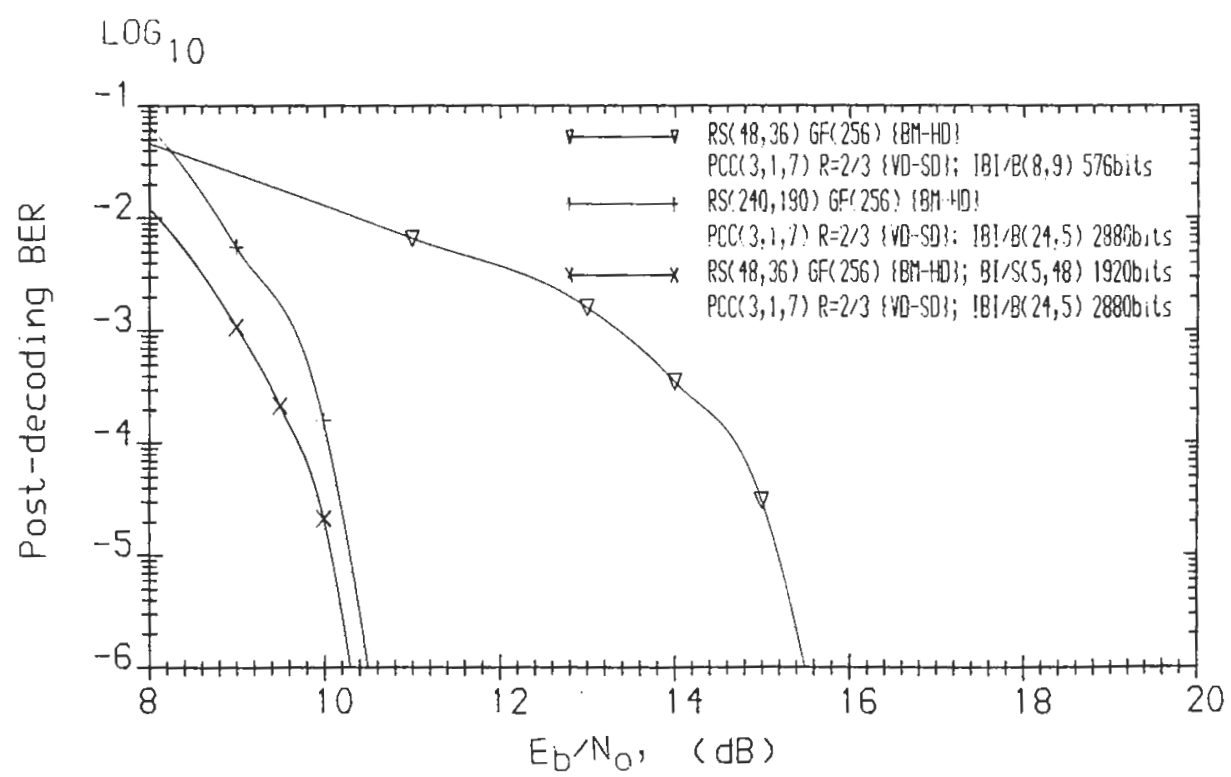

Figure 28. Post-decoding BER of concatenated code for data and speech transmission on a Rayleigh fading channel

outer and inner encoding positions. An appropriate arrangement is where an inner convolutional coder and an outer RS coder are used. As the inter-block interleaver has a high capability of randomizing the burst errors it is deployed as the inner interleaver for the convolutional coder. The outer interleaver is operating on RS code words and hence a block interleaver is selected having $n$ columns.

The choice of the convolutional coder as the inner coder is because it can deploy soft-decision Viterbi decoding, and has an ability to be punctured to a convenient coding rate. The output of the inner channel convolutional decoder at the receiver may contain burst errors, particularly when it is overloaded, and following symbol deinterleaving by the outer deinterleaver the bursts are distributed across a number of RS code words. This distribution is such as to enable the RS decoder to be generally successful in its error correction.
Proceeding on the basis that the inner and outer interleavers have effectively randomized the error bursts, we now determine the BER for the concatenated arrangement shown in Figure 1. To do this we calculate the post-decoding bit error rate $p_{\mathrm{bp}}$ for the convolutional decoder which we obtain from equation (6), and substitute it as $p_{b}$ in equation (31) to give the overall post-decoding bit error probability.

In our experimental arrangement the outer FEC codec in Figure 1 was an $\operatorname{RS}(240,180)$ or $\operatorname{RS}(48,36)$ code over $G F(256)$ that used hard-decision Berlekamp-Massey decoding, and the outer interleaver was not employed for the $\operatorname{RS}(240,180)$ code because of the relatively long code-word length. The inner FEC code was a $\operatorname{PCC}(3,1,7)$ 2/3-rate punctured code with Viterbi decoding by soft decision. The inner inter-block interleaver had $B$ $=24$ and $N=5$, yielding an interleaver delay of 
$2880 \mathrm{~b}$ its duration for our long-delay, high-integrity data channel, whereas the outer rectangular block interleaver, used in conjunction with the $\mathrm{RS}(48,36)$ code had the parameters $D=5$ and $W=48$. The overall coding rate was $\frac{1}{2}$, resulting in a transmitting rate of $32 \mathrm{~kb} / \mathrm{s}$, if $16 \mathrm{~kb} / \mathrm{s}$ input rate was assumed. Based on these parameters we derive two different long-delay, high-integrity channels, which we refer to as the data channel, having an interleaving period of 2880 bits. A short-delay speech channel, having an interleaving period of 576 bits is also introduced.

Figure 28 shows the variation of the post-decoding bit error probability, as a function of $E_{\mathrm{b}} / N_{\mathrm{o}}$ for the Rayleigh channel in case of three different coding schemes. Operating at an $E_{\mathrm{b}} / N_{0}$ in excess of $10 \mathrm{~dB}$ ensured that the BER was significantly below $10^{-6}$ in the case of the 2880 bit delay schemes. The overall delay of $180 \mathrm{~ms}$, computed at $16 \mathrm{~kb} / \mathrm{s}$ input transmission rate, is acceptable for data transmission. Observe that although the two codes have the same interleaving delay, the scheme with the $\operatorname{RS}(48,36)$ outer code with the rectangular outer block interleaver performs slightly better, owing to the randomizing effect of the outer interleaver deployed, although the $\operatorname{RS}(240,180)$ code is longer. The concatenated arrangement has a further advantage in that the RS outer codec has a powerful error detection capability, as opposed to the $\mathrm{CC}(2,1,7)$ convolutional codec. Namely, the $\operatorname{RS}(48,36)$ code has a relative error detection probability of 1 $10^{-9}$, whereas the corresponding value for the $\operatorname{RS}(240,180)$ code is effectively unity. For speech transmissions we suggest the scheme constituted by a $\operatorname{PCC}(3,1,7)$ inner code using soft-decision Viterbi decoding, as well as an $\operatorname{RS}(48,36)$ outer code combined with a 576 bits long inter-block interleaver. The performance of this scheme is more modest, according to its shorter delay of 576 bits, which corresponds to an overall coding delay of $36 \mathrm{~ms}$. The relative error-detection probability of this code has been stated to be $1-10^{-9}$, and it enables the RS outer codec to initiate post-enhancement techniques for improving speech quality. This approach has been followed in Reference 24.

Finally, we summarize our experiences in a condensed form to help the reader find his way through the extensive results section. Coding gain tables are presented as follows: for the nonconcatenated codes over the AWGN channel in Table III and over the Rayleigh-fading channel in Table IV; and for the concatenated codes over the Rayleigh-fading channel in Table $\mathrm{V}$. The first column describes the coding scheme used, the second and third columns contain the $E_{b} / N_{0}$ values for values of $\mathrm{BER}=10^{-3}$ and $\mathrm{BER}=10^{-6}$, whereas the third and fourth columns contain the actual coding gains at $\mathrm{BER}=10^{-3}$ and $\mathrm{BER}=10^{-6}$, respectively. These BER values are of prominent importance for data transmission and speech transmission, respectively.

We first compare a number of scenarios in terms of their coding gains at $B E R=10^{-6}$ over the Gaussian channel. It is remarkable that the $\mathrm{CC}(2,1,7)$ code has the best coding gain among the class of nonconcatenated codes over the AWGN channel. It has $1 \mathrm{~dB}$ better coding gain than the $\mathrm{CC}(2,1,5)$ code, which is attributed to its higher free distance. Surprisingly, the $\operatorname{PCC}(3,1,7)$ punctured code has nearly the same performance as the lower coding rate $\mathrm{CC}(2,1,7)$ code at approximately the same complexity. Whence, for most applications, the punctured code is preferred. The short RS codes have very low coding gains, whereas their long counterparts perform better, but not as well as the convolutional codes with soft-decision decoding. If we employ soft-decision trellis decoding for RS codes, the coding gains are improved, but the codeword length is very much limited by the exponentially increasing complexity.

Over Rayleigh-fading channels RS codes perform better than convolutional codes, when no interleaving is used, since they combat equally well both random and bursty errors. However, if interleaving is introduced, the situation is reversed, if the

Table III. Coding gain of non-concatenated codes over a Gaussian channel

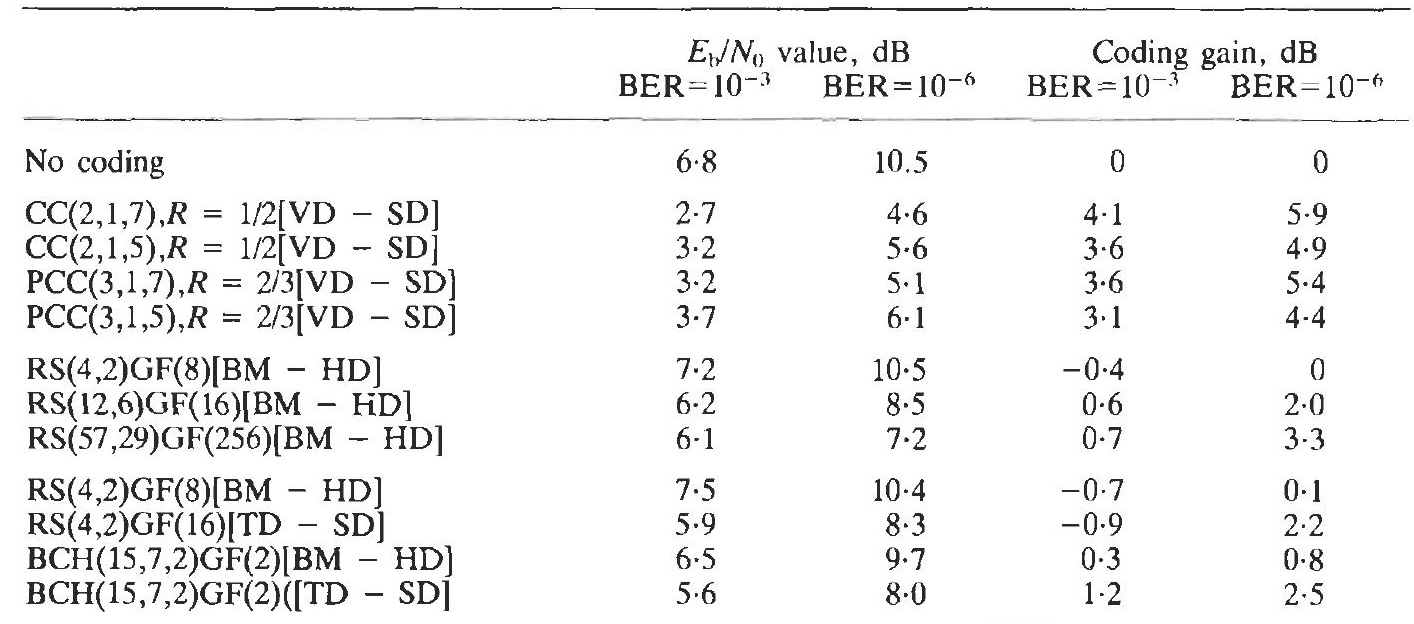


Table IV. Coding gain of non-concatenated codes over Rayleigh fading channel

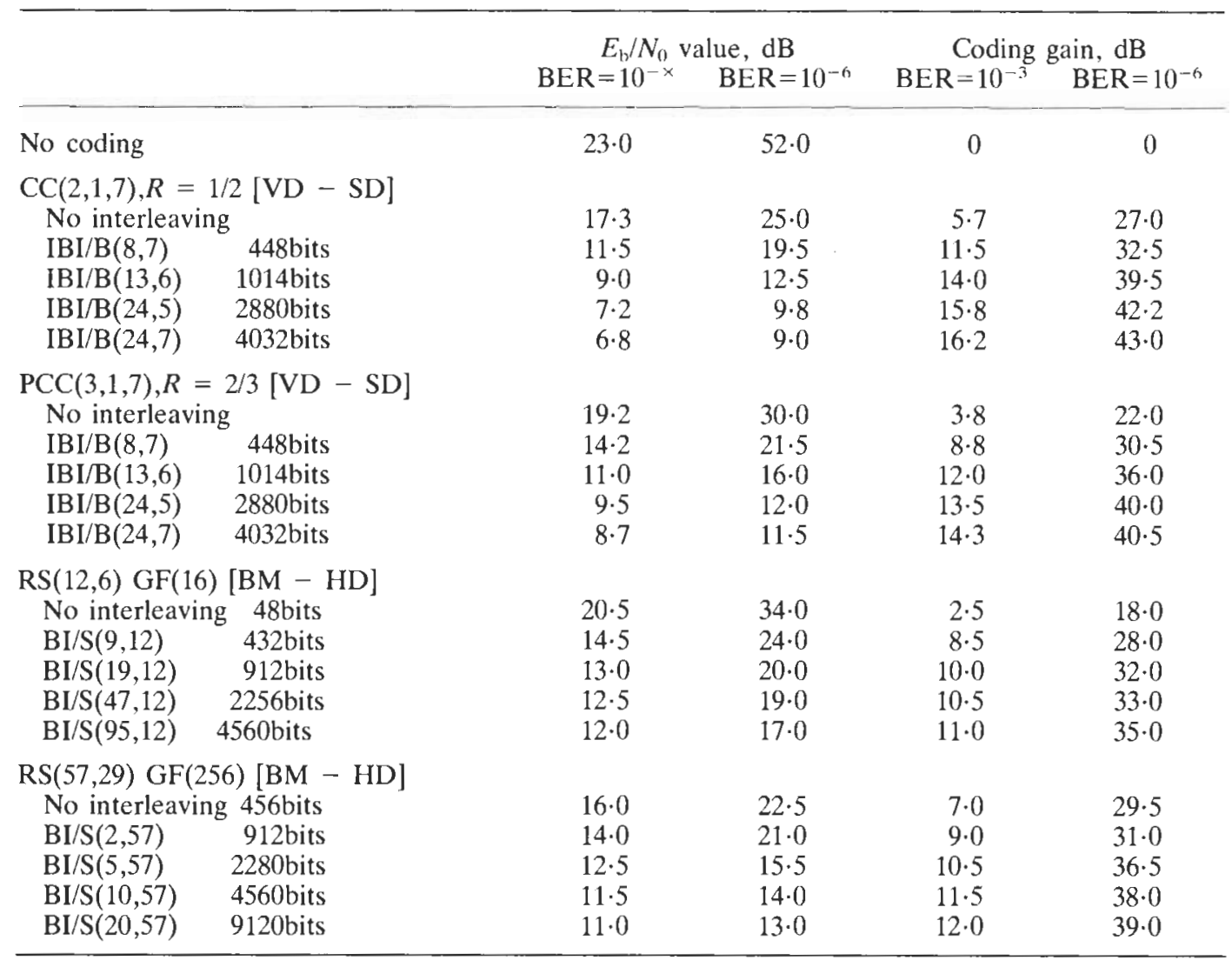

Table V. Coding gain of concatenated codes over Rayleigh fading channel

\begin{tabular}{|c|c|c|c|c|}
\hline & \multicolumn{2}{|c|}{$E_{\mathrm{b}} / N_{0}$ value, $\mathrm{dB}$} & \multicolumn{2}{|c|}{ Coding gain, dB } \\
\hline & $\mathrm{BER}=10^{-3}$ & $\mathrm{BER}=10^{-6}$ & $\mathrm{BER}=10^{-3}$ & $\mathrm{BER}=10^{-6}$ \\
\hline No coding & $23 \cdot 0$ & $52 \cdot 0$ & 0 & 0 \\
\hline $\begin{array}{l}\operatorname{RS}(48,36) \mathrm{GF}(256)[\mathrm{BM}-\mathrm{HD}] \\
\operatorname{PCC}(3,1,7), R=2 / 3[\mathrm{VD}-\mathrm{SD}] \\
\mathrm{IBI} / \mathrm{B}(8,9) \quad 576 \text { bits }\end{array}$ & $13 \cdot 3$ & $15 \cdot 5$ & $9 \cdot 7$ & $36 \cdot 5$ \\
\hline $\begin{array}{l}\mathrm{RS}(48,36) \mathrm{GF}(256)[\mathrm{BM}-\mathrm{HD}] \\
\mathrm{BI} / \mathrm{S}(5,48) \quad 1920 \mathrm{bits} \\
\mathrm{PCC}(3,1,7), R=2 / 3[\mathrm{VD}-\mathrm{SD}] \\
\mathrm{IBI} / \mathrm{B}(24,5) \quad 2880 \mathrm{bits}\end{array}$ & $9 \cdot 0$ & $10 \cdot 2$ & $14 \cdot 0$ & $41 \cdot 8$ \\
\hline $\begin{array}{l}\operatorname{RS}(240,180) \mathrm{GF}(256)[\mathrm{BM}-\mathrm{HD}] \\
\mathrm{PCC}(3,1,7), R=2 / 3[\mathrm{VD}-\mathrm{SD}] \\
\mathrm{IBI} / \mathrm{B}(24,5) \quad 2880 \mathrm{bits}\end{array}$ & $9 \cdot 5$ & $10 \cdot 5$ & $13 \cdot 5$ & $41 \cdot 5$ \\
\hline
\end{tabular}

interleaving memory of the convolutional code is set to the code-word length of the RS code.

In the case of concatenated codes the coding gain is approximately as high as in case of the nonconcatenated $C C(2,1,7)$ code, but at the price of slightly higher complexity, reliable error detection is achieved.

\section{CONCLUSIONS}

The performance of convolutional and ReedSolomon (RS) codes has been investigated both analytically and by simulation. The convolutional coder (CC) employed Viterbi decoding with soft decision, whereas the RS coder used either Berlekamp-Massey hard-decision decoding or softdecision trellis decoding. The interleaver selected for the RS coder was the rectangular block interleaver, and an inter-block interleaver was used in conjunction with the $\mathrm{CC}$. For the same coding rate concatenating the RS and $\mathrm{CC}$ coders was shown to have approximately the same coding gain as the $\mathrm{CC}$ coders on their own, but the concatenated code had the advantage of reliable error detections. The $\mathrm{CC}$ was employed as an inner coder in order to correct the burst errors randomized by the inter-block 
interleaver. However, when the $\mathrm{CC}$ was overloaded a burst error resulted. The outer de-interleaver working on a symbol basis distributed the errors such that the RS decoder had, in general, less than $t$ errors per block.

For speech communications the encoded rate of $16 \mathrm{~kb} / \mathrm{s}$ is concatenation coded to $32 \mathrm{~kb} / \mathrm{s}$, and the interleaving introduced an overall delay of $36 \mathrm{~ms}$. For transmissions over a Rayleigh fading channel, a BER $<10^{-3}$ was achieved from an $E_{\mathrm{b}} / N_{0}$ in excess of $13 \cdot 3 \mathrm{~dB}$. When computer data were transmitted, the interleaving period was increased to 2880 bits. We found that for a $\mathrm{BER}<10^{-6}$ an $E_{\mathrm{l}} / N_{0}$ value of $10 \cdot 2 \mathrm{~dB}$ was required, and that the probability of detecting an uncorrectable error was $1-10^{-9}$. The latter characteristic may be used to activate an automatic repeat request (ARQ) system.

\section{REFERENCES}

1. R. Steele, 'Towards a high-capacity digital cellular mobile radio system', IEE Proc., Pt F, 132, (5), 396-404 (1985).

2. J. A. Audestad, 'Network aspects of the GSM system', $8 t h$ European Conf. on Electrotechnics, Conf. Proc., on Area Commun., Sweden, June 1988, pp. 462-465.

3. P. Rasmussen, 'ISDN services', 8th European Conf. on Electrotechnics, Conf. Proc., on Area Commun., Sweden, June 1988, pp. 444447

4. A. Ghais, G. Berzins and D. Wright, 'INMARSAT and the future of mobile satellite services', IEEE J. Selected Areas Commun., SAC-5, (4), 592-600 (1987).

5. J. Hagenauer, F. Dolainsky, E. Lutz, W. Papke and R. Schweikert, 'The maritime satellite communication channel-channel model, performance of modulation and coding', IEEE J. Selecled Areas Commun., SAC-5, (4), 701-713 (1987).

6. D. J. Fang, F. T. Tseng and T. T. Calvit, 'A low elevation angle propagation measurement of $1.5 \mathrm{GHz}$ satellite signals in the Gulf of Mexico', IEEE Trans. Antenna Propagat., AP-30, (1), 10-15 (1982)

7. E. Lutz, W. Papke and E. Plöchinger, 'Land mobile satellite communications-channel model, modulation and error control', Proc. 7th Int. Conf. Digital Satellite Commun., Munich, May 1986.
8. GSM Recommendation 05.03, 'Channel coding', Draft Version 3.1.0, February 1988

9. A. J. Viterbi, 'Error bounds for convolutional codes and asymptotically optimum decoding algorithm', IEEE Trans. Info. Theory, IT-13, 260-269 (1967).

10. A. J. Viterbi, 'Convolutional codes and their performance in communication systems', IEEE Trans. Commun. Technol, COM-19, (5), 751-772 (1971).

11. C. G. Clark and J. B. Cain, Error-Correction Coding for Digital Communications, Plenum Press, New York, 1981.

12. E. R. Berlekamp, Algebraic Coding Theory, McGraw-Hill, 1968.

13. R. E. Blahut, Theory and Practice of Error Control Codes, Addison-Wesley, 1983.

14. J. K. Wolf, 'Efficient maximum likelihood decoding of linear block codes using a trellis', IEEE Trans. Info. Theory, IT24, (1), 76-80 (1978).

15. J. L. Ramsey, 'Realization of optimum interleavers', IEEE Trans. Info. Theory, IT-16, (3), 338-345 (1970).

16. K. Y. Liu and J. J. Lee, 'Recent results on the use of concatenated Reed-Solomon/Viterbi channel coding and data compression for space communications', IEEE Trans. Commun., COM-32, (5), 518-523 (1984)

17. K. J. Larsen, 'Short convolutional codes with maximal free distance for rate $1 / 2,1 / 3$ and $1 / 4$ ', IEEE Trans. Info. Theory, IT-19, 371-372 (1973).

18. K. H. H. Wong and R. Steele, "Transmission of digital speech in highway microcells', Journal of IERE, 57, (6) (supplement), S246-S254 (1987).

19. J. B. Cain, G. C. Clark and J. M. Geist, 'Punctured convolutional codes of rate $(n-1) / n$ and simplified maximum likelihood decoding', IEEE Trans. Info. Theory, IT-25, (1), 97-100 (1979).

20. Y. Yasuda, K. Kashiki and Y. Hirata, 'High-rate punctured convolutional codes for soft decision Viterbi decoding', IEEE Trans. Commun., COM-32, (3), 315-319 (1984).

21. A. M. Michelson and A. H. Levesque, Error-Control Techniques for Digital Communication, Wiley, 1985.

22. C. R. P. Hartmann and L. D. Rudolph, 'An optimum symbol-by-symbol decoding rule for linear codes', IEEE Trans. Info. Theory, IT-22, (5), 514-517 (1976).

23. L, R. Bahl, J. Cocke, F. Jelinek and J. Raviv, 'Optimum decoding of linear codes of minimizing symbol error rate', IEEE Trans. Info. Theory, IT-20, 284-287 (1974).

24. K. H. J. Wong, L. Hanzo and R. Steele, 'A sub-band codec with embedded Reed-Solomon coding for mobile radio speech communication', Procedings of ICCS 1988, Singapore, 31 October-3 November 1988. 